\title{
The WZNW model on $\operatorname{PSU}(1,1 \mid 2)$
}

\author{
Gerhard Götz ${ }^{1}$, Thomas Quella ${ }^{2,3}$, Volker Schomerus ${ }^{1,4}$ \\ 1 Service de Physique Théorique, CEA Saclay, \\ F-91191 Gif-sur-Yvette, France \\ 2 King's College London, Department of Mathematics, \\ Strand, London WC2R 2LS, United Kingdom \\ ${ }^{3} \mathrm{KdV}$ Institute for Mathematics, University of Amsterdam, \\ Plantage Muidergracht 24, 1018 TV Amsterdam, The Netherlands \\ 4 DESY Theory Group, DESY Hamburg, \\ Notkestrasse 85, D-22603 Hamburg, Germany
}

\begin{abstract}
According to the work of Berkovits, Vafa and Witten, the non-linear sigma model on the supergroup PSU $(1,1 \mid 2)$ is the essential building block for string theory on $\mathrm{AdS}_{3} \times \mathrm{S}^{3} \times \mathrm{T}^{4}$. Models associated with a non-vanishing value of the $\mathrm{RR}$ flux can be obtained through a psu $(1,1 \mid 2)$ invariant marginal deformation of the WZNW model on $\operatorname{PSU}(1,1 \mid 2)$. We take this as a motivation to present a manifestly $\operatorname{psu}(1,1 \mid 2)$ covariant construction of the model at the Wess-Zumino point, corresponding to a purely NSNS background 3-form flux. At this point the model possesses an enhanced $\widehat{\mathrm{psu}}(1,1 \mid 2)$ current algebra symmetry whose representation theory, including explicit character formulas, is developed systematically in the first part of the paper. The space of vertex operators and a free fermion representation for their correlation functions is our main subject in the second part. Contrary to a widespread claim, bosonic and fermionic fields are necessarily coupled to each other. The interaction changes the supersymmetry transformations, with drastic consequences for the multiplets of localized normalizable states in the model. It is only this fact which allows us to decompose the full state space into multiplets of the global supersymmetry. We analyze these decompositions systematically as a preparation for a forthcoming study of the RR deformation.
\end{abstract}

e-mail: Gerhard.Goetz@cea.fr, vschomer@mail.desy.de, tquella@science.uva.nl 


\section{Introduction}

String theory duals to superconformal field theories in various dimensions (see [1] for a review) can be related to 2D sigma models on supergroups and cosets (see [2, 3, 4, 5, 6, 6] for some early references). The precise relation depends on the particular framework, i.e. whether the models arise within the Green-Schwarz formalism, the hybrid or the pure spinor approach. These developments provide strong motivation to study world-sheet models with supermanifolds as target spaces. This applies in particular to $1+1$ dimensional sigma models on the superconformal group $\operatorname{PSU}(1,1 \mid 2)$. In this case, the hybrid formalism developed by Berkovits, Vafa and Witten [4] furnishes a covariant construction of string theory on $\mathrm{AdS}_{3} \times \mathrm{S}^{3}$. The main constituent of their formulation is a sigma model on $\operatorname{PSU}(1,1 \mid 2) \cdot 1$

Type IIB string theory on $\mathrm{AdS}_{3} \times \mathrm{S}^{3}$ has one rather peculiar feature, namely that the conditions on background fields imposed by the string equations of motion may be solved by both RR and/or NSNS 3-form fluxes. Hence, there exists a 2-parameter family of $\mathrm{AdS}_{3} \times \mathrm{S}^{3}$ backgrounds with an unbroken PSU $(1,1 \mid 2)$ symmetry. It is well known that models with pure NSNS background fields are easiest to deal with and indeed string theory in $\mathrm{AdS}_{3} \times \mathrm{S}^{3}$ has been solved for such cases using the NSR formalism [13, 14], based on earlier work on the Euclidean model [15, 16, 17]. According to common folklore, however, incorporating RR fluxes in the NSR formulation is conceptually difficult. This is where the hybrid approach comes in: it essentially removes the conceptual issues, but certainly leaves us with the hard task of solving the non-linear sigma model on $\operatorname{PSU}(1,1 \mid 2)$.

Though very little is known about sigma models on superspaces, there exist a few interesting results that are particularly relevant in our present context. Most importantly, Bershadsky et al. [18] have argued that quantum theories with $\operatorname{PSL}(N \mid N)$ target space are conformally invariant even before including the familiar WZ term. Of course the latter may then be added with any integer coefficient, preserving conformal invariance. Such a behavior can ultimately be traced back to the vanishing of the dual Coxeter number of $\operatorname{PSL}(N \mid N)$ along with the uniqueness of the invariant rank 3 tensor. This observation fits nicely with the before-mentioned parametrization of $\mathrm{AdS}_{3} \times \mathrm{S}^{3}$ backgrounds. WZNW models on $\operatorname{PSU}(1,1 \mid 2)$ at level $k$ describe pure NSNS backgrounds with $N=k+2$ units

\footnotetext{
${ }^{1}$ The same model has been proposed to describe plateau transitions in the integer quantum Hall effect [8] (see also [9, 10] for some further studies in this context). Let us note that models with superalgebra symmetries arise quite generally when systems with disorder are treated using Efetov's supersymmetric method [11] (see also [12] for a review).
} 
of NSNS flux running through the 3-sphere. Varying the coefficient of the kinetic relative to the WZ term corresponds to adding RR flux, see [4] for a precise relation between the parameters. Hence, the hybrid formulation offers a conceptually very simple description of $\mathrm{AdS}_{3} \times \mathrm{S}^{3}$ backgrounds with RR flux through marginal deformations of PSU $(1,1 \mid 2)$ WZNW models. Let us note that the parameter associated with RR fluxes is continuous in perturbative string theory since the mass of D5-branes is suppressed by a factor $g_{s}$ relative to the mass of NS5-branes.

Obviously, the construction of sigma models on $\operatorname{PSU}(1,1 \mid 2)$ through marginal deformation of the WZNW theory remains a very difficult technical problem. To begin with, surprisingly little is known even about WZNW models on supergroups. As we shall demonstrate below, models with current superalgebra symmetries behave very differently from their bosonic counterparts. The second obstacle arises with the RR deformation which is still technically hard to control since it breaks many of the local symmetries of the underlying world-sheet model. In fact, it was shown in [18] that switching on the deformation reduces the chiral symmetries of the conformal field theory from a full $\operatorname{psu}(1,1 \mid 2)$ current algebra to the chiral algebra generated by the so-called Casimir fields which is too small a symmetry to render the model solvable within a standard conformal field theory analysis 2 Nevertheless, some conformal field theory techniques, and in particular conformal perturbation theory, do offer a promising approach to computing certain spectra in the theory, even at generic points in the moduli space. We shall come back to this issue in a forthcoming paper.

The main focus of this work is on the $\operatorname{psu}(1,1 \mid 2)$ covariant construction of the WZNW model on the PSU $(1,1 \mid 2)$. We exploit and extend the insights which have been gained recently in [20] where the WZNW on GL(1|1) has been re-examined using a free field representation. In comparison to the earlier solution of the GL(1|1) WZNW model by Rozansky and Saleur [21], the new approach linked some of the peculiar properties of the field theory to characteristic features of super-geometry. In this geometric context, it can be argued in particular that WZNW models on supergroups give rise to examples of logarithmic conformal field theories (see e.g. [22, 23, 24, 25] and references therein). The appearance of logarithmic singularities had been observed repeatedly before in the theory of disordered systems (see e.g. [26, 27, 28]). Another property of the GL(1|1) WZNW

\footnotetext{
${ }^{2}$ In [18, the misnomer "Casimir algebra" was used for the generic chiral symmetry of the deformed models. This deviates from standard conventions. In fact, except for very special cases the Casimir algebra is much larger than the algebra of Casimir fields (see [19] for a nice review).
} 
model that was also established in [20] is its symmetry with respect to a special spectral flow automorphism of the current superalgebra. We shall encounter the same features in the PSU $(1,1 \mid 2)$ WZNW model, though the derivation is a bit different due to the noncompactness of the target space. The logarithmic singularities turn out to affect only the sector of localized normalizable states in the theory. It is a somewhat surprising outcome of this analysis that - contrary to a widespread believe, see e.g. 9] - the WZNW model on $\operatorname{PSU}(1,1 \mid 2)$ does not simply factorize into a product of the usual bosonic subsector and a bunch of free fermions. Such a factorization applies only to the free field theory that is used in the construction, but receives an interesting correction due to a non-trivial screening charge. The latter modifies, in particular, the transformation laws of fields in a rather non-trivial way. This fact becomes crucial for a successful RR deformation of the theory (see below and our forthcoming paper).

We have decided to separate the material of this paper into two parts. The first contains a rather complete discussion of the representation theoretic foundations for both the finite dimensional Lie algebra $\operatorname{psl}(2 \mid 2)$ and its affine version $\widehat{\operatorname{psl}}(2 \mid 2)_{k}$. Special attention is devoted to infinite dimensional representation of $\operatorname{psl}(2 \mid 2)$. Among the main new results are explicit character formulas for all irreducible representations of $\widehat{\operatorname{psl}}(2 \mid 2)_{k}$ belonging to finite dimensional representations and the infinite dimensional discrete and principal series. The second part then deals with the PSU $(1,1 \mid 2)$ WZNW model. After an extended discussion of the action functional, we study the state space first in the minisuperspace approximation. It is shown that the Laplacian on $\operatorname{PSU}(1,1 \mid 2)$ is non-diagonalizable, and the structure of the Jordan blocks is discussed. In fact, we shall provide explicit formulas for all its generalized eigenfunctions and study their transformation law wrt. the action of $\operatorname{psl}(2 \mid 2)$. Following this discussion, we explain how correlators of the WZNW model on $\operatorname{PSU}(1,1 \mid 2)$ can be computed starting from correlation functions for the WZNW model on the bosonic base. We shall also see how the non-trivial properties of the minisuperspace theory re-emerge in the field theory, giving rise to those features of the WZNW model we have outlined in the previous paragraph. Finally, as an application of our main results, we shall address the Casimir decomposition of the state space. More precisely, we describe an algorithm that allows to count all the states of the theory which transform in the same representation with respect to the global symmetries. These results shall serve as a starting point for a forthcoming analysis of the RR deformation. 


\section{Part I: Representation theory}

The first part of this work is devoted to the representation theory of both the finite dimensional Lie superalgebra $\operatorname{psl}(2 \mid 2)$ and its affine counterpart. We shall discuss finite and infinite dimensional representations of $\operatorname{psl}(2 \mid 2)$ and the corresponding modules of the $\operatorname{psl}(2 \mid 2)$ current algebra. Some results on the finite dimensional representations of psl(2|2) are fairly standard but they are included for completeness (see [29, 30, 31, 32] for more details and references). We believe that our analysis of representations of the affine algebra and their characters are new.

\section{Representation theory of $\operatorname{psl}(2 \mid 2)$}

In this section we shall discuss the Lie superalgebra psl(2|2) and its finite and infinite dimensional representations. The latter come in two series, namely a principal continuous and a 'discrete' series. We will not comment on the complementary series since it does not have any physical significance in the context we are interested in.

\subsection{The Lie superalgebra $\operatorname{psl}(2 \mid 2)$}

The Lie superalgebra $\mathfrak{g}=\operatorname{psl}(2 \mid 2)$ possesses six bosonic generators $K^{a b}=-K^{b a}$ with $a, b=1, \ldots, 4$. They form the Lie algebra so(4) which is isomorphic to $\mathfrak{g}^{(0)}=\operatorname{sl}(2) \oplus \operatorname{sl}(2)$. In addition, there are eight fermionic generators that we denote by $S_{\alpha}^{a}$. They split into two sets $(\alpha=1,2)$ each of which transform in the vector representation of so(4) $(a=1, \ldots, 4)$ which is the $(1 / 2,1 / 2)$ of $\operatorname{sl}(2) \oplus \operatorname{sl}(2)$. The relations of $\operatorname{psl}(2 \mid 2)$ are then given by

$$
\begin{aligned}
{\left[K^{a b}, K^{c d}\right] } & =i\left[\delta^{a c} K^{b d}-\delta^{b c} K^{a d}-\delta^{a d} K^{b c}+\delta^{b d} K^{a c}\right] \\
{\left[K^{a b}, S_{\gamma}^{c}\right] } & =i\left[\delta^{a c} S_{\gamma}^{b}-\delta^{b c} S_{\gamma}^{a}\right] \\
{\left[S_{\alpha}^{a}, S_{\beta}^{b}\right] } & =\frac{i}{2} \epsilon_{\alpha \beta} \epsilon^{a b c d} K^{c d} .
\end{aligned}
$$

Here, $\epsilon_{\alpha \beta}$ and $\epsilon^{a b c d}$ denote the usual completely antisymmetric $\epsilon$-symbols with $\epsilon_{12}=1$ and $\epsilon^{1234}=1$, respectively. An invariant metric is given by

$$
\left\langle K^{a b}, K^{c d}\right\rangle=-\epsilon^{a b c d} \quad\left\langle S_{\alpha}^{a}, S_{\beta}^{b}\right\rangle=-\epsilon_{\alpha \beta} \delta^{a b} .
$$


It is unique up to a scalar factor. The signs have been chosen in view of the real form $\operatorname{psu}(1,1 \mid 2)$ which will be considered below. In order to define a root space decomposition of $\operatorname{psl}(2 \mid 2)$ we split the fermions $\mathfrak{g}^{(1)}$ into two sets of four generators

$$
\mathfrak{g}_{+}^{(1)}=\operatorname{span}\left\{S_{1}^{a}\right\} \quad, \quad \mathfrak{g}_{-}^{(1)}=\operatorname{span}\left\{S_{2}^{a}\right\}
$$

As indicated by the subscripts \pm , we shall think of the fermionic generators $S_{1}^{a}$ as annihilation operators and of $S_{2}^{a}$ as creation operators.

In our discussion below we shall also employ a second basis which clearly exhibits the $\operatorname{sl}(2) \oplus \operatorname{sl}(2)$ structure of the bosonic subalgebra. The two Cartan generators of this new basis are given by

$$
K_{1}^{0}=\frac{1}{2}\left[K^{12}+K^{34}\right] \quad K_{2}^{0}=\frac{1}{2}\left[K^{12}-K^{34}\right] .
$$

These are supplemented by the bosonic raising and lowering operators of the form

$$
\begin{aligned}
& K_{1}^{ \pm}=\frac{1}{2}\left[K^{14}+K^{23} \pm i K^{24} \mp i K^{13}\right] \\
& K_{2}^{ \pm}=\frac{1}{2}\left[-K^{14}+K^{23} \mp i K^{24} \mp i K^{13}\right] .
\end{aligned}
$$

The elements $K_{\alpha}^{ \pm}$either commute with $K_{1}^{0}, K_{2}^{0}$ or shift the corresponding eigenvalue by \pm 1 . Finally there are four fermionic raising and four fermionic lowering operators $(\alpha=1,2)$

$$
S_{1 \alpha}^{ \pm}=S_{\alpha}^{1} \pm i S_{\alpha}^{2} \quad S_{2 \alpha}^{ \pm}=S_{\alpha}^{3} \pm i S_{\alpha}^{4}
$$

which raise/lower the eigenvalues of $K_{1}^{0}, K_{2}^{0}$ by $\pm 1 / 2$. A complete set of relations between the new generators of the Lie algebra $\operatorname{psl}(2 \mid 2)$ can be read off from (3.1)-(3.10) below.

\subsection{Kac modules and their characters}

In the present case the bosonic subalgebra $\mathfrak{g}^{(0)}$ consists of two commuting copies of $\operatorname{sl}(2)$. The Kac modules [33] of $\operatorname{psl}(2 \mid 2)$ are then labelled by pairs $(\mu, \nu)$ of representations $\mu, \nu$ of $s l(2)$. By construction, we declare that the corresponding representation space $V_{(\mu, \nu)}$ is annihilated by $S_{1}^{a}$ and then generate the Kac module $[\mu, \nu]$ through application of the raising operators $S_{2}^{a}$,

$$
[\mu, \nu]:=\operatorname{Ind}_{\mathfrak{g}^{(0)} \oplus \mathfrak{g}_{+}^{(1)}}^{\mathfrak{g}} V_{(\mu, \nu)}=\mathcal{U}(\mathfrak{g}) \otimes_{\mathfrak{g}^{(0)} \oplus \mathfrak{g}_{+}^{(1)}} V_{(\mu, \nu)}
$$


Here, we have extended the $\mathfrak{g}^{(0)}$-module $V_{(\mu, \nu)}$ to a representation of $\mathfrak{g}^{(0)} \oplus \mathfrak{g}_{+}^{(1)}$ by setting $S_{1}^{a} V_{(\mu, \nu)}=0$. Note that we can apply at most four fermionic generators to the states in $V_{(\mu, \nu)}$. When choosing the labels $\mu, \nu$ we silently agreed to identify the Cartan subalgebra of $\operatorname{psl}(2 \mid 2)$ with that of its maximal bosonic subalgebra.

To each of these Kac modules we can associate a supercharacter according to the standard prescription 3

$$
\chi_{[\mu, \nu]}\left(z_{1}, z_{2}\right)=\operatorname{str}\left[z_{1}^{K_{1}^{0}} z_{2}^{K_{2}^{0}}\right]=\operatorname{tr}\left[(-1)^{F} z_{1}^{K_{1}^{0}} z_{2}^{K_{2}^{0}}\right]=\chi_{\mu}\left(z_{1}\right) \chi_{\nu}\left(z_{2}\right) \chi_{F}\left(z_{1}, z_{2}\right) .
$$

It encodes the complete information about the weight content but not how the vectors are linked internally. The symbols $\chi_{\mu}$ and $\chi_{\nu}$ denote sl(2)-characters while the last factor $\chi_{F}$ stems from the contribution of the fermionic generators and is independent of the choice of $\mu$ and $\nu$. For the definition of the supercharacters we will always assume that the ground states, i.e. the states in the representation $V_{(\mu, \nu)}$ we started with, are bosonic.

In order to determine the fermionic term $\chi_{F}$ in the characters (2.6) we recall that the fermionic raising operators transform in the representation $(1 / 2,1 / 2)$ of $\operatorname{sl}(2) \oplus \operatorname{sl}(2)$ while products of more than one generator transform in antisymmetrized products thereof. This implies that the fermionic contribution to Kac modules has the bosonic content 4

$$
V_{F}=2(0,0) \oplus 2(1 / 2,1 / 2) \oplus(1,0) \oplus(0,1) .
$$

From this we read off immediately that

$$
\chi_{F}\left(z_{1}, z_{2}\right)=4+z_{1}+z_{1}^{-1}+z_{2}+z_{2}^{-1}-2\left(z_{1}^{\frac{1}{2}}+z_{1}^{-\frac{1}{2}}\right)\left(z_{2}^{\frac{1}{2}}+z_{2}^{-\frac{1}{2}}\right) .
$$

We introduced a special symbol for this representation since it will appear frequently throughout the text. Note that $\chi_{F}=\chi_{[0,0]}$ coincides with the character of the Kac module generated from the trivial representation.

Obviously, the bosonic contributions to the characters will strongly depend on the labels $\mu$ and $\nu$. In view of our applications to the Lie supergroup PSL(2|2) we are in fact primarily interested in representations for which $\nu=j_{2}=0,1 / 2,1, \ldots$, labels the finite dimensional irreducible representations of $\operatorname{sl}(2)$ so that

$$
\chi_{\nu}\left(z_{2}\right)=\chi_{j_{2}}\left(z_{2}\right)=\sum_{l=-j_{2}}^{j_{2}} z_{2}^{l} .
$$

\footnotetext{
${ }^{3} \mathrm{We}$ wish to emphasize that characters and supercharacters are related by the substitution $z^{1 / 2} \rightarrow$ $-z^{1 / 2}$. Hence, they both encode precisely the same information. Some of the formulas below possess a more natural interpretation in terms of supercharacters though.

${ }^{4}$ Here and in the following the phrase "bosonic content" refers to the decomposition of a Lie superalgebra module with respect to the maximal bosonic subalgebra.
} 
The first label $\mu$, on the other hand, will be allowed to run through three different series of representations.

Finite dimensional representations of $\operatorname{psl}(2 \mid 2)$ are obtained when we set $\mu=j_{1}=$ $0,1 / 2,1, \ldots$ In this case, the contribution to the characters (2.6) is given by $\chi_{\mu}\left(z_{1}\right)=$ $\chi_{j_{1}}\left(z_{1}\right)$ as defined in (2.9). Even though such representations are not associated to unitary representations of $\mathrm{su}(1,1) \oplus \mathrm{su}(2)$ unless $j_{1}=j_{2}=0$, finite dimensional representations play an important role, in particular for the boundary WZNW model.

Our second series of $\operatorname{psl}(2 \mid 2)$ representations is affiliated with the two discrete series of $\operatorname{su}(1,1)$. In this case, the label is $\mu=\left( \pm, j_{1}\right)$ with $j_{1}<0$ any negative real number 5 With our choice of $j_{1}$ and of the indefinite metric (2.2), the value of the Casimir element in $\left( \pm, j_{1}\right)$ is given by $-j_{1}\left(j_{1}+1\right)$. By definition, the representations $\left(+, j_{1}\right)$ and $\left(-, j_{1}\right)$ have a lowest/highest weight with $K_{1}^{0}$ eigenvalues $-j_{1}>0$ and $j_{1}<0$, respectively. The corresponding characters are given by

$$
\chi_{\left( \pm, j_{1}\right)}\left(z_{1}\right)=\sum_{n=0}^{\infty} z_{1}^{\mp j_{1} \pm n}=\frac{z_{1}^{\mp j_{1}}}{1-z_{1}^{ \pm 1}} .
$$

In the last two lines the geometric series expression is valid for $\left|z_{1}\right|<1$ and $\left|z_{1}\right|>1$, respectively. Let us emphasize again that, in our conventions, the representations $\left(+, j_{1}\right)$ are actually labelled by a negative real number $j_{1}$ although their lowest weight has a positive weight $-j_{1}$.

The last set of representations we need comes with the principal continuous series of $\operatorname{su}(1,1)$. We label such representations by tupels $\mu=\left(j_{1}, \alpha\right)$ where $0 \leq \alpha<1$ and $j_{1} \in \mathbb{S}=-1 / 2+i \mathbb{R}$. Representations in the principal continuous series have neither highest nor lowest weight states. Eigenvalues of the Cartan element $K_{1}^{0}$ take values on $\alpha+\mathbb{Z}$. Hence the characters of the third series read

$$
\chi_{\left(j_{1}, \alpha\right)}\left(z_{1}\right)=\sum_{n \in \mathbb{Z}} z_{1}^{\alpha+n}
$$

Note that these characters do not depend on the spin $j_{1}$. Yet, the latter determines the value $-j_{1}\left(j_{1}+1\right)$ of the quadratic Casimir.

The importance of Kac modules stems from the fact that they are irreducible for generic values of the labels $\mu$ and $\nu$. Nevertheless, for special atypical choices of $(\mu, \nu)$, nontrivial invariant subspaces exist. A close inspection of the action of fermionic generators on

\footnotetext{
5 Our notation seems to deviate from the standard one but it appears to be closer to the actual construction of the modules and hence the formulas we encounter will be easier.
} 
Kac modules reveals that, starting with a bosonic highest or lowest weight representation, there is just one single atypicality condition which may be written in the form 6

$$
0=\left(j_{2}-j_{1}\right)\left(j_{1}+j_{2}+1\right)=-j_{1}\left(j_{1}+1\right)+j_{2}\left(j_{2}+1\right)=C_{2}\left(j_{1}, j_{2}\right),
$$

i.e. the Kac module $[\mu, \nu]$ possesses a non-trivial invariant submodule if and only if the quadratic Casimir of the bosonic subalgebra vanishes on the multiplet $(\mu, \nu)$ from which the Kac module is generated. For finite dimensional representations this happens whenever $j_{1}=j_{2}$. Similarly, the Kac modules $\left[\left( \pm, j_{1}\right), j_{2}\right]$ cease to be irreducible if and only if $j_{1}=-j_{2}-1$. In the following we shall study the atypical cases in much more detail.

In case of the principal continuous series, finally, the atypically condition (2.12) does not apply. But since the value of the quadratic Casimir is directly determined by the label of the bosonic highest weight multiplet and identical on the whole representation generated from it we can give a necessary condition for the decoupling of a bosonic subrepresentation $\left(\left(j_{1}^{\prime}, \alpha^{\prime}\right), j_{2}^{\prime}\right)$ of the Kac module $\left[\left(j_{1}, \alpha\right), j_{2}\right]$ : The eigenvalues of the Casimir operator have to agree. A careful analysis of this condition including the discussion of possible decomposition series then shows that the Kac modules $\left[\left(j_{1}, \alpha\right), j_{2}\right]$ are always irreducible.

\subsection{Finite dimensional atypical representations}

As described in the previous subsection, finite dimensional Kac modules of $\operatorname{psl}(2 \mid 2)$ are labelled by pairs $\left[j_{1}, j_{2}\right]$ with $j_{i}=0,1 / 2,1, \ldots$ A Kac module $\left[j_{1}, j_{2}\right]$ is irreducible whenever $j_{1} \neq j_{2}$. In case $j_{1}=j_{2}$, however, Kac modules turn out to be indecomposable composites of smaller irreducible building blocks (short multiplets). We shall describe the latter in the next paragraph before discussing the new class of so-called projective covers. These are maximal indecomposable composites of short multiplets. In some sense that we shall make more precise below, the projective covers should be considered as the natural replacement of Kac modules in case we are dealing with atypical representations.

\subsubsection{Atypical Kac modules and irreducible representations}

As we have mentioned several times, the Kac modules $[j, j]$ contain non-trivial invariant subspaces. For $j \geq 1$ the structure of the Kac module can be encoded in the following chain

$$
[j, j]: \quad[j] \rightarrow\left[j+\frac{1}{2}\right] \oplus\left[j-\frac{1}{2}\right] \rightarrow[j],
$$

\footnotetext{
${ }^{6}$ This conditions arises if one tries to return from the state $S_{2}^{1} S_{2}^{2} S_{2}^{3} S_{2}^{4}\left|j_{1}, j_{2}\right\rangle$ on the fourth fermion level to the original highest weight state $\left|j_{1}, j_{2}\right\rangle$.
} 
where $[j]$ denote irreducible atypical representations (short multiplets) of $\operatorname{psl}(2 \mid 2)$. The structure of the reducible Kac modules can also be depicted by a planar diagram in which the vertical direction refers to the spin $j$ of the atypical constituents,

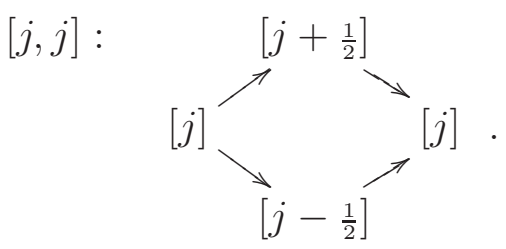

Since pictures of this type will appear frequently throughout this text, let us pause here for a moment and explain carefully how to decode their information. We read the diagram (2.13) from right to left. The rightmost entry in our chain contains the so-called socle of the indecomposable representation, i.e. the largest fully reducible invariant submodule we can find. In the case of our Kac module, the socle happens to be irreducible and it is given by the atypical representation $[j]$. If we divide the Kac module by the submodule $[j]$, we obtain a new indecomposable representation of our Lie superalgebra. Its diagram is obtained from the one above by removing the last entry and all arrows connected to it. The socle of this quotient is a direct sum of the two atypical representations $[j \pm 1 / 2]$. It is rather obvious how to iterate this procedure until the entire indecomposable representation is split up into floors with only direct sums of irreducible representations appearing on each floor.

There are two special cases for which the decomposition of the Kac module does not follow the generic pattern as described in eq. (2.13). These are the cases $j=0$ and $j=1 / 2$,

$$
\begin{array}{ll}
{[0,0]:} & {[0] \rightarrow\left[\frac{1}{2}\right] \rightarrow[0]} \\
{\left[\frac{1}{2}, \frac{1}{2}\right]:} & {\left[\frac{1}{2}\right] \rightarrow[1] \rightarrow[0] \oplus[0] \rightarrow\left[\frac{1}{2}\right] .}
\end{array}
$$

The irreducible constituents [0] and [1/2] are the trivial one-dimensional representation and the 14-dimensional adjoint representation of $\operatorname{psl}(2 \mid 2)$.

The supercharacters of short multiplets can be deduced from those of the corresponding Kac modules and the composition patterns (2.13), (2.15) and (2.16). They possess the form

$$
\chi_{[j]}\left(z_{1}, z_{2}\right)=2 \chi_{j}\left(z_{1}\right) \chi_{j}\left(z_{2}\right)-\chi_{j+\frac{1}{2}}\left(z_{1}\right) \chi_{j-\frac{1}{2}}\left(z_{2}\right)-\chi_{j-\frac{1}{2}}\left(z_{1}\right) \chi_{j+\frac{1}{2}}\left(z_{2}\right)
$$


for all $j>0$. We would like to stress that these supercharacters do not contain the fermionic factor $\chi_{F}$ that appears in all supercharacters of typical irreducible representations.

\subsubsection{Projective covers of $[j]$}

In the previous subsection we have seen the first examples of representations which are built out of several short multiplets. Kac modules are only one example of such composites and we shall indeed need another class of indecomposables as we proceed, the so-called projective covers $\mathcal{P}_{j}$. By definition, these are the largest indecomposables whose socle consists of a single atypical representation $[j]$. General results imply that such a maximal indecomposable extension of $[j]$ exists and is unique [29]. In case of $j \geq 3 / 2$, the structure of $\mathcal{P}_{j}$ is encoded in the following diagram

$$
\begin{aligned}
\mathcal{P}_{j}:[j] \longrightarrow 2\left[j+\frac{1}{2}\right] \oplus 2\left[j-\frac{1}{2}\right] & \longrightarrow[j+1] \oplus 4[j] \oplus[j-1] \longrightarrow \\
\longrightarrow 2\left[j+\frac{1}{2}\right] \oplus 2\left[j-\frac{1}{2}\right] \longrightarrow[j] &
\end{aligned}
$$

Note that $\mathcal{P}_{j}$ contains an entire Kac module as a proper submodule. In this sense, the Kac modules are extendable. We also observe one rather generic feature of projective covers: they are built up from different Kac modules in a way that resembles the pattern in which Kac modules are constructed out of irreducibles (see eq. (2.13) $)$.7 One may see this even more clearly if $\mathcal{P}_{j}$ is displayed as a 2-dimensional diagram in which the additional direction keeps track of the spin $j$ of the atypical constituents $[j]$,

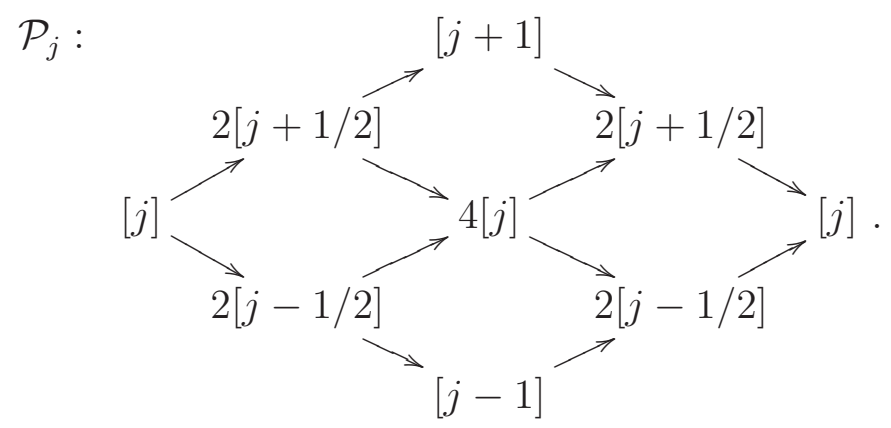

We will continue to switch between such planar pictures and diagrams of the form (2.18). The remaining cases $j=0,1 / 2,1$ have to be listed separately. When $j=1$ the picture is very similar only that we have to insert $2[0]$ in place of $[j-1]$,

$$
\mathcal{P}_{1}:[1] \longrightarrow 2\left[\frac{3}{2}\right] \oplus 2\left[\frac{1}{2}\right] \longrightarrow[2] \oplus 4[1] \oplus 2[0] \longrightarrow 2\left[\frac{3}{2}\right] \oplus 2\left[\frac{1}{2}\right] \longrightarrow[1]
$$

\footnotetext{
${ }^{7}$ In mathematics this statement is known as a generalization of the BGG duality theorem [29].
} 
The projective cover of the atypical representation $[1 / 2]$ is obtained from the generic case by the formal substitution $2[j-1 / 2] \rightarrow 3[0]$,

$$
\mathcal{P}_{\frac{1}{2}}:\left[\frac{1}{2}\right] \longrightarrow 2[1] \oplus 3[0] \longrightarrow\left[\frac{3}{2}\right] \oplus 4\left[\frac{1}{2}\right] \longrightarrow 2[1] \oplus 3[0] \longrightarrow\left[\frac{1}{2}\right]
$$

Finally, the projective cover $\mathcal{P}_{0}$ of the trivial representation is given by,

$$
\mathcal{P}_{0}:[0] \longrightarrow 3\left[\frac{1}{2}\right] \longrightarrow 2[1] \oplus 6[0] \longrightarrow 3\left[\frac{1}{2}\right] \longrightarrow[0]
$$

The reader is invited to convert the last three formulas into planar pictures.

This concludes our list of the projective covers of finite dimensional representations. The representations $\mathcal{P}_{j}$ appear in the operator products of certain open string vertex operators in the WZNW model, when we consider boundary conditions corresponding to a point-like brane. Together, typical representations and the projective covers of atypicals form the subset of so-called projective representations. What makes this class particularly interesting is its behavior under tensor products. In fact, it is well-known that projective representations of a Lie superalgebra form an ideal in the fusion ring. This means that the product of a projective representation with any other representation, no matter how complicated it is, can be decomposed into projectives. We shall later see that this property of projective representations (along with the fact that they are easy to list) has invaluable consequences.

It is moreover relevant to observe that, unlike for the atypicals themselves, the characters of their projective covers contain the full fermionic character $\chi_{F}$ as a factor. To be precise one has

$$
\chi_{\mathcal{P}_{j}}=\left[2 \chi_{j}\left(z_{1}\right) \chi_{j}\left(z_{2}\right)-\chi_{j+\frac{1}{2}}\left(z_{1}\right) \chi_{j+\frac{1}{2}}\left(z_{2}\right)-\chi_{\left|j-\frac{1}{2}\right|}\left(z_{1}\right) \chi_{\left|j-\frac{1}{2}\right|}\left(z_{2}\right)\right] \chi_{F}\left(z_{1}, z_{2}\right) .
$$

This property puts projective covers on an equal footing with typical irreducibles. Eventually, it will even allow us to come up with a version of the familiar Racah-Speiser algorithm which holds for projective representations of Lie superalgebras. We refer readers interested in further details to section 7.1 below.

\subsection{Infinite dimensional atypical representations}

Let us now turn to the theory of infinite dimensional atypical representations of $\operatorname{psl}(2 \mid 2)$. As we have remarked before, atypicals appear only in the discrete series and if the labels $(\mu, \nu)=\left(\left( \pm, j_{1}\right), j_{2}\right)$ satisfy the condition $j_{1}+j_{2}+1=0$. The plan of this subsection 
follows the same logic as our discussion of finite dimensional atypicals, i.e. we shall study atypical Kac modules and irreducibles first and then turn to the projective covers. But since some of the results below seem to be less known, we will be a bit more detailed about their derivation.

\subsubsection{Atypical Kac modules and irreducible representations}

Kac modules of the form $[( \pm,-j-1), j]$ fail to be irreducible. In order to understand the structure of the resulting Kac modules let us first have a look at the bosonic content of typical representations,

$$
\left.[( \pm,-j-1), j]\right|_{\mathrm{sl}(2) \oplus \mathrm{sl}(2)}=(( \pm,-j-1), j) \otimes V_{F},
$$

where $V_{F}$ denotes the fermionic contributions as specified in (2.7). This tensor product can be evaluated using the familiar rules for $s l(2)$ and the additional formula

$$
( \pm,-j-1) \otimes k=\bigoplus_{l=-j-1-k}^{-j-1+k}( \pm, l)
$$

which holds as long as $j \geq 0$ and $k \leq j$, or more generally as long as the sum on the right hand side does not contain contributions with non-negative half-integer or integer $l$. It is straightforward to see that in $[( \pm,-j-1), j]$ the bosonic representations $(( \pm,-j-1), j)$, $\left(\left( \pm,-j-\frac{1}{2}\right), j-\frac{1}{2}\right)$ and $\left(\left( \pm,-j-\frac{3}{2}\right), j+\frac{1}{2}\right)$ decouple. After dividing out the induced invariant submodules we are left with the irreducible representation $[j]_{ \pm}$whose bosonic content reads

$$
\left.[j]_{ \pm}\right|_{\mathrm{sl}(2) \oplus \mathrm{sl}(2)}=2(( \pm,-j-1), j) \oplus\left(\left( \pm,-j-\frac{3}{2}\right), j-\frac{1}{2}\right) \oplus\left(\left( \pm,-j-\frac{1}{2}\right), j+\frac{1}{2}\right)
$$

for $j \neq 0$. We also infer that the structure of the degenerate Kac modules is described by the composition series

$$
[( \pm,-j-1), j]: \quad[j]_{ \pm} \rightarrow\left[j+\frac{1}{2}\right]_{ \pm} \oplus\left[j-\frac{1}{2}\right]_{ \pm} \rightarrow[j]_{ \pm} .
$$

Again, we assumed that $j \neq 0$. Formally this formula is identical to the one which is obtained for finite dimensional representations [32].

So far we have avoided to investigate the special case $j=0$. The characters of the irreducible representations $[0]_{ \pm}$are easily obtained from the above since these representations arise as building blocks of the Kac modules $[( \pm,-3 / 2), 1 / 2]$,

$$
\left.[0]_{ \pm}\right|_{\mathrm{sl}(2) \oplus \operatorname{sl}(2)}=2(( \pm,-1), 0) \oplus(( \pm,-1 / 2), 1 / 2) .
$$


Concerning the structure of the special Kac modules $[( \pm,-1), 0]$ we note that their bosonic content is given by

$$
\begin{aligned}
{\left.[( \pm,-1), 0]\right|_{\mathrm{sl}(2) \oplus \operatorname{sl}(2)}=} & 2(( \pm,-1), 0) \oplus 2(( \pm,-3 / 2), 1 / 2) \oplus 2(( \pm,-1 / 2), 1 / 2) \\
& \oplus \mathcal{R}_{(( \pm,-1), 0)} \oplus(( \pm,-2), 0) \oplus(( \pm,-1), 1)
\end{aligned}
$$

Let us stress that it contains an indecomposable bosonic representation $\mathcal{R}_{(( \pm,-1), 0)}$ which has the decomposition series

$$
\mathcal{R}_{(( \pm,-1), 0)}: \quad(( \pm,-1), 0) \rightarrow(0,0) \rightarrow(( \pm,-1), 0)
$$

The structure of the Kac module may be summarized in the decomposition series

$$
[( \pm,-1), 0]: \quad[0]_{ \pm} \rightarrow[0] \oplus[1 / 2]_{ \pm} \rightarrow[0]_{ \pm}
$$

It is interesting to find a finite dimensional representation in the decomposition series even though we started with an infinite dimensional representation.

For later applications we shall draw an important conclusion from the decomposition formulas (2.27) and (2.31) of Kac modules. Note that they allow us to express the supercharacter of the atypical trivial representation [0] formally as in infinite sum over supercharacters of Kac modules, 8

$$
\chi_{[0]}\left(z_{1}, z_{2}\right)=\sum_{n=0}^{\infty}(n+1) \chi_{[( \pm,-n / 2-1), n / 2]}\left(z_{1}, z_{2}\right) .
$$

Indeed, one can show by straightforward direct computation that the terms on the right hand side sum up to $\chi_{[0]}=1$. If the supercharacters of the Kac modules are decomposed into a sum of bosonic characters as encoded in formulas (2.27) and (2.31), then all but the contribution from the trivial representation cancel each other.

\subsubsection{Projective covers of $[j]_{ \pm}$}

In case of finite dimensional representations, the projective covers are built up from Kac modules and there exists a rather simple rule to determine the number of Kac modules of any type within a given projective cover [29]. If we extrapolate this rule to our present

\footnotetext{
${ }^{8}$ We have first learned this trick and its generalization to affine supercharacters from Hubert Saleur 34, see also 35, for a very simple version thereof.
} 
setup, we arrive at the following composition series for the projective covers of the discrete representations

$$
\begin{gathered}
\mathcal{P}_{j}^{ \pm}:[j]_{ \pm} \longrightarrow 2\left[j+\frac{1}{2}\right]_{ \pm} \oplus 2\left[j-\frac{1}{2}\right]_{ \pm} \longrightarrow[j+1]_{ \pm} \oplus 4[j]_{ \pm} \oplus[j-1]_{ \pm} \\
\longrightarrow 2\left[j+\frac{1}{2}\right]_{ \pm} \oplus 2\left[j-\frac{1}{2}\right]_{ \pm} \longrightarrow[j]_{ \pm}
\end{gathered}
$$

for $j \geq 1$. The same expression may be used for $j=1 / 2$ if we formally replace $\left[-\frac{1}{2}\right]_{ \pm}$ by the trivial representation [0]. The structure of the projective covers of $[0]_{ \pm}$does not follow the generic pattern. Instead it is given by

$$
\mathcal{P}_{0}^{ \pm} \quad: \quad[0]_{ \pm} \longrightarrow[0] \oplus 2\left[\frac{1}{2}\right]_{ \pm} \longrightarrow 3[0]_{ \pm} \oplus[1]_{ \pm} \longrightarrow[0] \oplus 2\left[\frac{1}{2}\right]_{ \pm} \longrightarrow[0]_{ \pm}
$$

Needless to stress that the characters of these projective covers contain the factor $\chi_{F}$ as in the finite dimensional case. 


\section{Representation theory of the affine superalgebra}

Irreducible representations of the affine $\operatorname{psl}(2 \mid 2)$ superalgebra can be built over all the irreducible representations of the $\operatorname{psl}(2 \mid 2)$ algebra that we discussed above. The latter can be either finite or infinite dimensional. We shall address the infinite dimensional ones first and then turn to the finite dimensional representations in the second subsection.

\subsection{The affine $\operatorname{psl}(2 \mid 2)$ algebra and spectral flows}

Here we display the definition of the current algebra $\widehat{\operatorname{psl}}(2 \mid 2)_{k}$ first. In terms of raising and lowering operators (2.3)-(2.5) it may be written as

$$
\begin{aligned}
{\left[K_{1, m}^{0}, K_{1, n}^{ \pm}\right] } & = \pm K_{1, m+n}^{ \pm} & {\left[K_{2, m}^{0}, K_{2, n}^{ \pm}\right] } & = \pm K_{2, m+n}^{ \pm} \\
{\left[K_{1, m}^{0}, S_{1 \alpha, n}^{ \pm}\right] } & = \pm \frac{1}{2} S_{1 \alpha, m+n}^{ \pm} & {\left[K_{1, m}^{0}, S_{2 \alpha, n}^{ \pm}\right] } & = \pm \frac{1}{2} S_{2 \alpha, m+n}^{ \pm} \\
{\left[K_{2, m}^{0}, S_{1 \alpha, n}^{ \pm}\right] } & = \pm \frac{1}{2} S_{1 \alpha, m+n}^{ \pm} & {\left[K_{2, m}^{0}, S_{2 \alpha, n}^{ \pm}\right] } & =\mp \frac{1}{2} S_{2 \alpha, m+n}^{ \pm} \\
\left\{S_{1 \alpha, m}^{ \pm}, S_{2 \beta, n}^{ \pm}\right\} & =\mp 2 \epsilon_{\alpha \beta} K_{1, m+n}^{ \pm} & \left\{S_{1 \alpha, m}^{ \pm}, S_{2 \beta, n}^{\mp}\right\} & = \pm 2 \epsilon_{\alpha \beta} K_{2, m+n}^{ \pm} \\
{\left[K_{1, m}^{ \pm}, S_{1 \alpha, n}^{\mp}\right] } & = \pm S_{2 \alpha, m+n}^{ \pm} & {\left[K_{1, m}^{ \pm}, S_{2 \alpha, n}^{\mp}\right] } & =\mp S_{1 \alpha, m+n}^{ \pm} \\
{\left[K_{2, m}^{ \pm}, S_{1 \alpha, n}^{\mp}\right] } & = \pm S_{2 \alpha, m+n}^{\mp} & {\left[K_{2, m}^{ \pm}, S_{2 \alpha, n}^{ \pm}\right] } & =\mp S_{1 \alpha, m+n}^{ \pm} .
\end{aligned}
$$

In addition, there are six relations involving the level $k$ of the $\operatorname{psl}(2 \mid 2)$ current algebra. These read as follows,

$$
\begin{array}{rlrl}
{\left[K_{1, m}^{0}, K_{1, n}^{0}\right]} & =-\frac{k}{2} m \delta_{m+n, 0} & {\left[K_{2, m}^{0}, K_{2, n}^{0}\right]=\frac{k}{2} m \delta_{m+n, 0}} \\
{\left[K_{1, m}^{+}, K_{1, n}^{-}\right]} & =2 K_{1, m+n}^{0}-m k \delta_{m+n, 0} & {\left[K_{2, m}^{+}, K_{2, n}^{-}\right]=2 K_{2, m+n}^{0}+m k \delta_{m+n, 0}} \\
\left\{S_{1 \alpha, m}^{+}, S_{1 \beta, n}^{-}\right\} & =2 \epsilon_{\alpha \beta}\left(K_{1, m+n}^{0}-K_{2, m+n}^{0}\right)-2 m k \epsilon_{\alpha \beta} \delta_{m+n, 0} \\
\left\{S_{2 \alpha, m}^{+}, S_{2 \beta, n}^{-}\right\} & =2 \epsilon_{\alpha \beta}\left(K_{1, m+n}^{0}+K_{2, m+n}^{0}\right)-2 m k \epsilon_{\alpha \beta} \delta_{m+n, 0}
\end{array}
$$

The algebra defined by eqs. (3.1) - (3.10) possesses a two-parameter family $\gamma^{\left(w_{1}, w_{2}\right)}$ of automorphisms. It is induced from the following two-parameter family of automorphisms for the bosonic subalgebra $\widehat{\mathrm{sl}}(2)_{-k} \oplus \widehat{\mathrm{sl}}(2)_{k}$

$$
\begin{array}{ll}
\gamma^{\left(w_{1}, w_{2}\right)}\left(K_{1, n}^{0}\right)=K_{1, n}^{0}-\frac{k}{2} w_{1} \delta_{n 0} & \gamma^{\left(w_{1}, w_{2}\right)}\left(K_{1, n}^{ \pm}\right)=K_{1, n \pm w_{1}}^{ \pm} \\
\gamma^{\left(w_{1}, w_{2}\right)}\left(K_{2, n}^{0}\right)=K_{2, n}^{0}+\frac{k}{2} w_{2} \delta_{n 0} & \gamma^{\left(w_{1}, w_{2}\right)}\left(K_{2, n}^{ \pm}\right)=K_{2, n \pm w_{2}}^{ \pm}
\end{array}
$$


One may easily check that these maps extend to the whole algebra $\widehat{\mathrm{psl}}(2 \mid 2)_{k}$ through

$$
\gamma^{\left(w_{1}, w_{2}\right)}\left(S_{1 \alpha, n}^{ \pm}\right)=S_{1 \alpha, n \pm \frac{w_{1}+w_{2}}{2}}^{ \pm} \quad \gamma^{\left(w_{1}, w_{2}\right)}\left(S_{2 \alpha, n}^{ \pm}\right)=S_{2 \alpha, n \pm \frac{w_{1}-w_{2}}{2}}^{ \pm} .
$$

Application of these automorphisms maps representations of the current algebra onto each other. They therefore play a crucial role in the representations theory of $\widehat{\operatorname{psl}}(2 \mid 2)_{k}$.

\subsubsection{Free field construction of the affine algebra}

Following [36, 4] we can construct $\widehat{\operatorname{psl}}(2 \mid 2)_{k}$ out of four pairs of fermions $p^{a}$ (of weight $h=1$ ) and $\theta^{a}$ (with $h=0$ ) which satisfy

$$
p^{a}(z) \theta^{b}(w)=\frac{\delta^{a b}}{z-w}
$$

and the affine $\operatorname{sl}(2)_{-k-2} \oplus \operatorname{sl}(2)_{k-2}$ algebra that is generated by currents $j^{a b}$ with the following operator product expansion

$$
j^{a b}(z) j^{c d}(w)=-\frac{k \epsilon^{a b c d}+2\left(\delta^{a c} \delta^{b d}-\delta^{a d} \delta^{b c}\right)}{(z-w)^{2}}+\frac{i\left[\delta^{a c} j^{b d}-\delta^{a d} j^{b c}-\delta^{b c} j^{a d}+\delta^{b d} j^{a c}\right]}{z-w} .
$$

Based on this structure we now obtain the $\widehat{\operatorname{psl}}(2 \mid 2)_{k}$ algebra via

$$
\begin{aligned}
K^{a b} & =j^{a b}-i\left[\theta^{a} p^{b}-\theta^{b} p^{a}\right], \quad S_{2}^{a}=p^{a} \\
S_{1}^{a} & =k \partial \theta^{a}+\frac{i}{2} \epsilon^{a b c d}\left[\theta^{b} j^{c d}-i \theta^{b} \theta^{c} p^{d}\right] .
\end{aligned}
$$

It is straightforward to show that the fields $K$ and $S_{\alpha}^{a}$ obey the correct relations following from eqs. (2.1) and (2.2).

One of the main ingredients in the representation theory of $\widehat{\operatorname{psl}}(2 \mid 2)_{k}$ are the spectral flow automorphisms which have been defined in eqs. (3.11)-(3.13). We would like to show that the spectral flows of the full affine Lie superalgebra are inherited from those which may be defined for $\operatorname{sl}(2)_{-k-2} \oplus \operatorname{sl}(2)_{k-2}$ and the fermions $p_{a}$ and $\theta^{a}$. For the bosonic current algebra we have the standard transformations

$$
\begin{array}{ll}
\gamma^{\left(w_{1}, w_{2}\right)}\left(J_{1, n}^{0}\right)=J_{1, n}^{0}-\frac{k+2}{2} w_{1} \delta_{n 0} & \gamma^{\left(w_{1}, w_{2}\right)}\left(J_{1, n}^{ \pm}\right)=J_{1, n \pm w_{1}}^{ \pm} \\
\gamma^{\left(w_{1}, w_{2}\right)}\left(J_{2, n}^{0}\right)=J_{2, n}^{0}+\frac{k-2}{2} w_{2} \delta_{n 0} & \gamma^{\left(w_{1}, w_{2}\right)}\left(J_{2, n}^{ \pm}\right)=J_{2, n \pm w_{2}}^{ \pm}
\end{array}
$$

In view of the schematic structure $K=J+(p \theta)$ of the currents appearing in the full superalgebra we also need to implement a non-trivial transformation on the fermions. A 
suitable basis is

$$
\begin{array}{ll}
p_{1}^{ \pm}=p^{1} \pm i p^{2}=S_{12}^{ \pm} & p_{2}^{ \pm}=p^{3} \pm i p^{4}=S_{22}^{ \pm} \\
\theta_{1}^{ \pm}=\theta^{1} \pm i \theta^{2} & \theta_{2}^{ \pm}=\theta^{3} \pm i \theta^{4}
\end{array}
$$

Starting from the defining relation $\left\{p_{m}^{a}, \theta_{n}^{b}\right\}=\delta^{a b} \delta_{m+n, 0}$ there remain the following nonvanishing anti-commutators

$$
\left\{p_{1, m}^{ \pm}, \theta_{1, n}^{\mp}\right\}=2 \delta_{m+n, 0} \quad\left\{p_{2, m}^{ \pm}, \theta_{2, n}^{\mp}\right\}=2 \delta_{m+n, 0}
$$

Employing (3.11) and (3.13), a natural and consistent candidate for the spectral flow on the fermions is

$$
\begin{array}{ll}
\gamma^{\left(w_{1}, w_{2}\right)}\left(p_{1, n}^{ \pm}\right)=p_{1, n \pm \frac{w_{1}+w_{2}}{2}}^{ \pm} & \gamma^{\left(w_{1}, w_{2}\right)}\left(p_{2, n}^{ \pm}\right)=p_{2, n \pm \frac{w_{1}-w_{2}}{2}}^{ \pm} \\
\gamma^{\left(w_{1}, w_{2}\right)}\left(\theta_{1, n}^{ \pm}\right)=\theta_{1, n \pm \frac{w_{1}+w_{2}}{2}}^{ \pm} & \gamma^{\left(w_{1}, w_{2}\right)}\left(\theta_{2, n}^{ \pm}\right)=\theta_{2, n \pm \frac{w_{1}-w_{2}}{2}}^{ \pm}
\end{array}
$$

One can easily check that this transformation leaves (3.21) invariant. The consistency of the action of $\gamma^{\left(w_{1}, w_{2}\right)}$ on $j^{a b}$ and the fermions $p$ and $\theta$ with the spectral flow on $K_{1, n}^{ \pm}, K_{1, n}^{ \pm}$, $K_{1, m}^{0}$ and $K_{2, m}^{0}$ given in (3.11) and (3.12) can be verified by expressing the latter in terms of the $\left(p^{ \pm}, \theta^{ \pm}\right)$-basis and taking care about normal ordering.

\subsection{Typical representations and their supercharacters}

In the following we shall be concerned with the various representations of the affine algebra $\widehat{\operatorname{psl}}(2 \mid 2)_{k}$. Without further mentioning we shall always assume that the level $k$ is an integer $k \geq 3$. The standard representations are obtained by acting with generators of negative mode number $n$ on ground states which can transform in the various representations of the zero mode algebra $\operatorname{psl}(2 \mid 2)$. Hence, generic representations $[\mu, \nu]^{\wedge}$ of the current algebra are labelled by the same pairs of $\operatorname{sl}(2)$ representation labels $\mu, \nu$ as the modules $[\mu, \nu]$ of $\operatorname{psl}(2 \mid 2)$, with some additional $k$-dependent truncations on the possible range of the spin labels.

As before we take the second label $\nu=j_{2}=0,1 / 2,1, \ldots$ to be a half-integer. We shall also require that $j_{2} \leq k / 2-1$. As for the first label, there are again three different series corresponding to $\mu=\left( \pm, j_{1}\right)$ (for $-\frac{1}{2}>j_{1}>-\frac{k+1}{2}$ ) or $\mu=\left(j_{1}, \alpha\right)$ (for $j_{1} \in \mathbb{S}=$ $\left.-\frac{1}{2}+i \mathbb{R}\right)$ for representations with an infinite number of ground states and to $\mu=j_{1}=$ 
$0,1 / 2,1, k / 2+1$ when the number of ground states is finite. In terms of these labels, the ground states of the associated representations possess conformal dimension 9

$$
h_{\left[\left( \pm, j_{1}\right), j_{2}\right]}=\left(-j_{1}\left(j_{1}+1\right)+j_{2}\left(j_{2}+1\right)\right) / k
$$

Our aim is to describe the singular vectors in the Verma modules and to provide the associated formulas for the supercharacters

$$
\chi_{R}\left(q, z_{1}, z_{2}\right):=\operatorname{str}_{R}\left(q^{L_{0}-\frac{c}{24}} z_{1}^{K_{1}^{0}} z_{2}^{K_{2}^{0}}\right)
$$

of irreducible representations since these are the basic building blocks of any type of representation. We define typical representations to be those which result from Verma modules in which all the singular vectors are inherited from the bosonic subalgebra. As a consequence, these typical representations have a very nice representation in terms of the free field construction (3.16). In order to clarify this statement we recall that the pairs of fermions have a unique representation $\mathcal{F}$ if we restrict ourselves to integer moding 10 Given any irreducible representation $\mathcal{V}_{(\mu, \nu)}$ of $\widehat{\mathrm{sl}}(2)_{-k-2} \oplus \widehat{\mathrm{sl}}(2)_{k-2}$ we may then define an action of $\widehat{\operatorname{psl}}(2 \mid 2)_{k}$ on the generalized Fock module

$$
\mathcal{V}_{[\mu, \nu]}=\mathcal{V}_{(\mu, \nu)} \otimes \mathcal{F}
$$

using the free fermion realization. Our terminology ensures that this representation is irreducible if and only if it is typical. This follows from the analysis of the Kac-Kazhdan determinant, see appendix A. Hence, the supercharacter of an irreducible typical representation takes the form

$$
\begin{gathered}
\chi_{[\mu, \nu]}\left(q, z_{1}, z_{2}\right)=\frac{1}{\eta^{4}(q)} \prod_{a, b= \pm 1} \vartheta_{1}\left(z_{1}^{a / 2} z_{2}^{b / 2}, q\right) \chi_{\mu}^{-k-2}\left(q, z_{1}\right) \chi_{\nu}^{k-2}\left(q, z_{2}\right) \\
\text { where } \vartheta_{1}(y, q)=-i y^{1 / 2} q^{1 / 8} \prod_{n=1}^{\infty}\left(1-q^{n}\right)\left(1-y q^{n}\right)\left(1-y^{-1} q^{n-1}\right) .
\end{gathered}
$$

The first factors $\vartheta_{1} / \eta$ in the character (3.25) arise from the four pairs of fermionic fields $p_{a}$ and $\theta^{a}$ in the free field construction.

Atypical Kac modules are obtained when $j_{1}-j_{2}=n k$ or $j_{1}+j_{2}+1=n k$ for some $n \in \mathbb{Z}$ and they possess additional singular vectors resulting from the application of fermionic

\footnotetext{
${ }^{9}$ We will omit the hat in affine representations if this interpretation is clear from the context.

${ }^{10}$ With non-integer moding global supersymmetry cannot be realized in the WZNW model we are aiming at.
} 
generators, see appendix A. Given the physical $k$-dependent bounds on the spins it is easy to see that the only atypicality conditions which apply are $j_{1}=j_{2}$ and $j_{1}+j_{2}+1=0$, just as in the zero mode sector. Hence all the affine submodules originate from singular vectors on the level of the ground states. The corresponding irreducible representations of $\widehat{\operatorname{psl}}(2 \mid 2)_{k}$ are denoted by $[j]^{\wedge}$ and $[j]_{ \pm}^{\wedge}$, respectively. In the following we shall present explicit formulas for the supercharacters of all these representations.

\subsection{Representations with an infinite number of ground states}

To begin with we shall present formulas for the supercharacters of the discrete and principal continuous series representations of the affine algebra $\widehat{\operatorname{psl}}(2 \mid 2)_{k}$. In the former series special attention will be paid to the atypical cases. In this sector the characters may be expressed in terms of infinite sums of characters of typical representations, see [35] for the first formula of this type in the context of $\mathrm{gl}(1 \mid 1)$ and unpublished work of Hubert Saleur [34] for a more elaborate application.

\subsubsection{Typical discrete series representations}

Physically relevant typical representations of the discrete series are labelled by $j_{1}$ and $j_{2}$ with $j_{1}+j_{2}+1 \neq 0$ and $j_{1}<-1 / 2$. We shall also keep our restriction to values $j_{1} \geq-(k+$ 1)/2. Furthermore, $j_{2}$ are certainly taken from the usual set $j_{2}=0,1 / 2,1, \ldots, k / 2-1$. The characters of the corresponding representations of $\widehat{\operatorname{psl}}(2 \mid 2)_{k} \operatorname{read}$,

$$
\chi_{\left[\left( \pm, j_{1}\right), j_{2}\right]}\left(q, z_{1}, z_{2}\right)=\frac{1}{\eta^{4}(q)} \prod_{\nu, \mu= \pm} \vartheta_{1}\left(z_{1}^{\nu / 2} z_{2}^{\mu / 2}, q\right) \chi_{\left( \pm, j_{1}\right)}^{-k-2}\left(q, z_{1}\right) \chi_{j_{2}}^{k-2}\left(q, z_{2}\right)
$$

where $j_{1}+j_{2}+1 \neq 0$. Let us recall that the relevant $\widehat{\mathrm{sl}}(2)$ characters are given by

$$
\begin{aligned}
\chi_{\left( \pm, j_{1}\right)}^{-k-2}\left(q, z_{1}\right) & = \pm i q^{-\frac{\left(j_{1}+1 / 2\right)^{2}}{k}} z_{1}^{\mp\left(j_{1}+1 / 2\right)} \vartheta_{1}\left(q, z_{1}\right)^{-1} \\
\chi_{j_{2}}^{k-2}\left(q, z_{2}\right) & =i q^{\frac{\left(j_{2}+1 / 2\right)^{2}}{k}} z_{2}^{j_{2}+1 / 2} \vartheta_{1}\left(q, z_{2}\right)^{-1} \Psi_{j_{2}}^{k}\left(q, z_{2}\right) \\
\text { where } \Psi_{j_{2}}^{k}\left(q, z_{2}\right) & =\sum_{a \in \mathbb{Z}} q^{k a^{2}+2 a\left(j_{2}+1 / 2\right)}\left(z_{2}^{a k}-z_{2}^{-a k-2\left(j_{2}+1 / 2\right)}\right) .
\end{aligned}
$$

We amend eq. (3.28) by the prescription to expand the function $\vartheta$ in powers of $z_{1}$ for the positive sign and in powers of $1 / z_{1}$ for the negative sign. Similarly, an expansion in $1 / z_{2}$ is to be used in equation (3.29). In the following we shall use the symbol $\left[\left( \pm, j_{1}\right), j_{2}\right]^{\wedge}$ for these irreducible representations of the affine algebra $\widehat{\operatorname{psl}}(2 \mid 2)_{k}$. 


\subsubsection{Atypical discrete series representations}

Nothing keeps us from evaluating the characters we introduced in the previous subsection at the special points where $j_{1}+j_{2}+1=0$. At these points, the Verma modules over the representations $[( \pm,-j-1), j]$ develop new fermionic singular vectors. The latter are all to be found among the ground states so that the composition of the atypical module from irreducibles is identical to the one for the corresponding Kac modules of the horizontal subalgebra $\operatorname{psl}(2 \mid 2)$. For the characters this implies

$$
\begin{aligned}
\chi_{[( \pm, j+1), j]}\left(q, z_{1}, z_{2}\right) & =2 \chi_{[j]_{ \pm}}\left(q, z_{1}, z_{2}\right)-\chi_{[j+1 / 2]_{ \pm}}\left(q, z_{1}, z_{2}\right)-\chi_{[j-1 / 2]_{ \pm}}\left(q, z_{1}, z_{2}\right) \\
\chi_{[( \pm, 1), 0]}\left(q, z_{1}, z_{2}\right) & =2 \chi_{[0]_{ \pm}}\left(q, z_{1}, z_{2}\right)-\chi_{[1 / 2]_{ \pm}}\left(q, z_{1}, z_{2}\right)-\chi_{[0]}\left(q, z_{1}, z_{2}\right) .
\end{aligned}
$$

Note that the character $\chi_{[0]}$ that appears in the last line is the character of the vacuum representation. The formulas (3.31) can be used to solve for the characters of atypical representations from the discrete series. In fact, formula (2.32) suggests that

$$
\begin{aligned}
\chi_{[j]_{ \pm}}\left(q, z_{1}, z_{2}\right) & =-\sum_{n=0}^{\infty}(n+1) \chi_{[( \pm,-(j+3 / 2+n / 2)), j+1 / 2+n / 2]}\left(q, z_{1}, z_{2}\right) \\
& = \pm \frac{1}{\eta^{4}(q) \vartheta_{1}\left(q, z_{1}\right) \vartheta_{1}\left(q, z_{2}\right)} \prod_{\nu, \mu= \pm} \vartheta_{1}\left(z_{1}^{\nu / 2} z_{2}^{\mu / 2}, q\right) \Psi_{j}^{ \pm}\left(q, z_{1}, z_{2}\right) \\
\text { with } \Psi_{j}^{ \pm}\left(q, z_{1}, z_{2}\right) & =\sum_{a \in \mathbb{Z}} q^{k a^{2}+2 a(j+1)}\left(\frac{z_{1}^{ \pm j \pm 1} z_{2}^{a k+j+1}}{\left(1-q^{a} z_{1}^{ \pm 1 / 2} z_{2}^{1 / 2}\right)^{2}}-\frac{z_{1}^{ \pm j \pm 1} z_{2}^{-a k-(j+1)}}{\left(1-q^{a} z_{1}^{ \pm 1 / 2} z_{2}^{-1 / 2}\right)^{2}}\right) .
\end{aligned}
$$

One may check that these characters indeed obey the relations (3.31). In view of the equation (2.32) we can also formally use the previous formula for the value $j=-1 / 2$ to determine the vacuum character of the $\operatorname{psl}(2 \mid 2)$ current algebra

$$
\begin{aligned}
\chi_{[0]}\left(q, z_{1}, z_{2}\right) & =-\frac{1}{\eta^{4}(q) \vartheta_{1}\left(q, z_{1}\right) \vartheta_{1}\left(q, z_{2}\right)} \prod_{\nu, \mu= \pm} \vartheta_{1}\left(z_{1}^{\nu / 2} z_{2}^{\mu / 2}, q\right) \Psi_{0}\left(q, z_{1}, z_{2}\right) \\
\text { with } \Psi_{0} & =\sum_{a \in \mathbb{Z}} q^{k a^{2}+a} z_{1}^{-1 / 2}\left(\frac{z_{2}^{a k+1 / 2}}{\left(1-q^{a} z_{1}^{-1 / 2} z_{2}^{1 / 2}\right)^{2}}-\frac{z_{2}^{-a k-1 / 2}}{\left(1-q^{a} z_{1}^{-1 / 2} z_{2}^{-1 / 2}\right)^{2}}\right) .
\end{aligned}
$$

This should be considered as an expansion in $1 / z_{1}$ and $1 / z_{2}$. Note that $\Psi_{0}=\Psi_{-1 / 2}^{+}=$ $\Psi_{-1 / 2}^{-}$. The functions $\Psi$ can be expressed as a derivative of higher level Appell functions [37], as in the case of $\operatorname{sl}(2 \mid 1)$ [38]. For an explicit evaluation we proceed as follows: We first divide the sum over $a \in \mathbb{Z}$ in two domains, $a \geq 0$ and $a<0$. For $a \geq 0$ we expand the denominator as it stands while for $a<0$ we first multiply both numerator 
and denominator with $q^{-2 a}$ in order to obtain an expansion in positive powers of $q$. The resulting expressions have been verified to reproduce the structure of singular vectors of the module $[0]^{\wedge}$ for $k=1,2, \ldots, 7$ and energies smaller or equal to $h=4$.

The formulas we have proposed also pass some more general non-trivial consistency checks. To begin with there is a simple relation between the characters of representations from the discrete series,

$$
\chi_{\left[\left( \pm, j_{1}\right), j_{2}\right]}\left(q, z_{1}, z_{2}\right)=\chi_{\left[\left(\mp, j_{1}\right), j_{2}\right]}\left(q, z_{1}^{-1}, z_{2}\right), \quad \chi_{[j]_{ \pm}}\left(q, z_{1}, z_{2}\right)=\chi_{[j]_{\mp}}\left(q, z_{1}^{-1}, z_{2}\right)
$$

This property expresses a manifest symmetry of the corresponding representations. In fact, under the reflection $K_{1}^{0} \rightarrow-K_{1}^{0}$ and an corresponding action on the fermions which promotes this transformation into an automorphism, the representations from the two different discrete series are mapped onto each other.

Our second crucial observation concerns the behavior of the characters under spectral flow. We consider the spectral flow automorphisms $\gamma_{ \pm}=\gamma^{(\mp 1,1)}$. Note that these generate all spectral flows that do not interpolate between Ramond and Neveu-Schwarz representations, i.e. that map integer mode numbers to integer mode numbers. On the zero modes $L_{0}, K_{1}^{0} \equiv K_{1,0}^{0}$ and $K_{2}^{0} \equiv K_{2,0}^{0}$ they act according to

$$
\gamma_{ \pm}\left(L_{0}\right)=L_{0}+K_{2}^{0} \mp K_{1}^{0} \quad, \quad \gamma_{ \pm}\left(K_{1}^{0}\right)=K_{1}^{0} \pm k / 2 \quad, \quad \gamma_{ \pm}\left(K_{2}^{0}\right)=K_{2}^{0}+k / 2 .
$$

On characters of representations, the action of the spectral flow can be expressed as

$$
\gamma_{ \pm}\left(\chi_{*}\left(q, z_{1}, z_{2}\right)\right)=z_{1}^{ \pm k / 2} z_{2}^{k / 2} \chi_{*}\left(q, q^{\mp 1} z_{1}, q z_{2}\right)
$$

It is rather easy to see that this action is consistent with the following behavior of irreducible representations,

$$
\left[\left( \pm, j_{1}\right), j_{2}\right]^{\wedge} \stackrel{\gamma_{\mp}}{\longrightarrow}\left[\left(\mp,-k / 2-1-j_{1}, k / 2-1-j_{2}\right]^{\wedge} \quad, \quad[j]_{ \pm}^{\wedge} \stackrel{\gamma_{\mp}}{\longrightarrow}[k / 2-1-j]_{\mp}^{\wedge} .\right.
$$

Combining all these observations we obtain the following equation in the case that the level $k$ is even

$$
z_{1}^{k / 2} z_{2}^{k / 2} \chi_{[k / 4-1 / 2]_{-}}\left(q, q^{-1} z_{1}, q z_{2}\right)=\chi_{[k / 4-1 / 2]_{+}}\left(q, q^{-1} z_{1}, q z_{2}\right)=\chi_{[k / 4-1 / 2]_{-}}\left(q, z_{1}^{-1}, z_{2}\right)
$$

A short computation shows that our formula (3.32) for the characters of discrete series representations provides a function $\chi_{[k / 4-1 / 2]_{-}}$with the desired property. This constitutes a rather strong test for the expression we proposed. 


\subsubsection{The continuous series representations}

As we have discussed above, the Lie superalgebra psl(2|2) possesses another type of infinite dimensional irreducible representations, the principal continuous series. These represen-

tations are labelled by pairs $\left[\left(j_{1}, \alpha\right), j_{2}\right]^{\wedge}$ where $j_{1} \in \mathbb{S}=1 / 2+i \mathbb{R}$ and $0 \leq \alpha<1$. These representations give rise to typical representations of the affine Lie superalgebra. Their characters are,

$$
\begin{aligned}
\chi_{\left[\left(j_{1}, \alpha\right), j_{2}\right]}\left(q, z_{1}, z_{2}\right) & =\frac{1}{\eta^{4}(q)} \prod_{\nu, \mu= \pm} \vartheta_{1}\left(z_{1}^{\nu / 2} z_{2}^{\mu / 2}, q\right) \chi_{\left(j_{1}, \alpha\right)}^{-k-2}\left(q, z_{1}\right) \chi_{j_{2}}^{k-2}\left(q, z_{2}\right) \\
\text { where } \chi_{\left(j_{1}, \alpha\right)}^{-k-2}\left(q, z_{1}\right) & =-i q^{-\frac{\left(j_{1}-1 / 2\right)^{2}}{k}} \chi_{(j, \alpha)}\left(z_{1}\right)\left(z_{1}^{1 / 2}-z_{1}^{-1 / 2}\right) \vartheta_{1}\left(q, z_{1}\right)^{-1} .
\end{aligned}
$$

These characters will be the most important building blocks for the partition sum of the bulk PSU(1, 1|2) WZNW model.

\subsection{Representations with a finite number of ground states}

Finally, we turn to representations with finite number of ground states. These do not appear in the bulk spectrum of the PSU $(1,1 \mid 2)$ WZNW model but are expected to furnish the building block for the boundary spectra associated with instantonic branes. Special attention is devoted to the atypical representations.

\subsubsection{Typical representations}

The free field construction we have reviewed above suggests that generic representations have no singular vectors except from the ones that arise through the representations of the two bosonic $\widehat{\operatorname{sl}}(2)$ current algebra at levels $\pm k-2$. This is indeed the case. The statement implies a precise expression for the characters of typical representations

$$
\chi_{\left[j_{1}, j_{2}\right]}\left(q, z_{1}, z_{2}\right)=\frac{1}{\eta^{4}(q)} \prod_{\nu, \mu= \pm} \vartheta_{1}\left(q, z_{1}^{\nu / 2} z_{2}^{\mu / 2}\right) \chi_{j_{1}}^{-k-2}\left(q, z_{1}\right) \chi_{j_{2}}^{k-2}\left(q, z_{2}\right)
$$

where $j_{1} \neq j_{2}$ and $j_{1} \leq k / 2+1$. The function $\vartheta_{1}$ was defined in eq. (3.25) above. We also recall that the $\widehat{\mathrm{sl}}(2)$ characters for negative level are given by

$$
\chi_{j_{1}}^{-k-2}\left(q, z_{1}\right)=i q^{-\frac{\left(j_{1}+1 / 2\right)^{2}}{k}}\left(z_{1}^{j_{1}+1 / 2}-z_{1}^{-j_{1}-1 / 2}\right) \vartheta_{1}\left(q, z_{1}\right)^{-1}
$$

We shall use the symbol $\left[j_{1}, j_{2}\right]^{\wedge}$ for these irreducible representations of the affine algebra. 


\subsubsection{Atypical representations}

Nothing prevents us from evaluating the previous character formulas at the points $j_{1}=$ $j=j_{2}$. But the resulting functions turn out to be the characters of indecomposable representations $[j, j]^{\wedge}$ which contain fermionic singular multiplets. The latter lie all within the space of ground states and hence the decomposition follows exactly the decomposition formulas (2.13|2.15]2.16) for Kac modules of the Lie superalgebra psl $(2 \mid 2)$

$$
\begin{aligned}
\chi_{[j, j]}\left(q, z_{1}, z_{2}\right) & =2 \chi_{[j]}\left(q, z_{1}, z_{2}\right)-\chi_{[j+1 / 2]}\left(q, z_{1}, z_{2}\right)-\chi_{[j-1 / 2]}\left(q, z_{1}, z_{2}\right) \\
\chi_{[1 / 2,1 / 2]}\left(q, z_{1}, z_{2}\right) & =2 \chi_{[1 / 2]}\left(q, z_{1}, z_{2}\right)-\chi_{[1]}\left(q, z_{1}, z_{2}\right)-2 \chi_{[0]}\left(q, z_{1}, z_{2}\right) \\
\chi_{[0,0]}\left(q, z_{1}, z_{2}\right) & =2 \chi_{[0]}\left(q, z_{1}, z_{2}\right)-\chi_{[1 / 2]}\left(q, z_{1}, z_{2}\right) .
\end{aligned}
$$

In the first row we assumed $j \geq 1$. The relative sign between the two terms on the right hand side is due to the fermionic nature of the singular vectors. Even though equation (3.38) is not a closed formula for the characters of atypical representations, it can be used to construct the latter recursively as a sum of the functions $\chi_{[j, j]}$ and of the vacuum character $\chi_{[0]}$. We have determined the latter in the previous subsection and hence know all characters, at least implicitly.

We can do a little better, though, and provide explicit expressions for the atypical characters. To this end we observe that the typical representations obey

$$
\chi_{\left[j_{1}, j_{2}\right]}\left(q, z_{1}, z_{2}\right)=\chi_{\left[\left(-, j_{1}+1\right), j_{2}\right]}\left(q, z_{1}, z_{2}\right)-\chi_{\left[\left(+, j_{1}+1\right), j_{2}\right]}\left(q, z_{1}, z_{2}\right)
$$

where we silently agreed to formally expand both characters on the right hand side in powers of $z_{1}$. Using this observation, we deduce that the characters of atypical representations $[j]^{\wedge}, j \geq 1 / 2$, must be given by

$$
\begin{aligned}
\chi_{[j]}\left(q, z_{1}, z_{2}\right) & =-\sum_{n=0}^{\infty}(n+1) \chi_{[j+1 / 2+n / 2, j+1 / 2+n / 2]}\left(q, z_{1}, z_{2}\right) \\
& =\chi_{[j]_{+}}\left(q, z_{1}, z_{2}\right)-\chi_{[j]_{-}}\left(q, z_{1}, z_{2}\right) \quad \text { for } \quad j \geq \frac{1}{2} .
\end{aligned}
$$

It is somewhat cumbersome but rather straightforward to check that these characters indeed obey the relations (3.38). Note that formula (3.39) should only be used for $j \geq 1 / 2$. The vacuum character is not given by expression (3.39) but rather by formula (3.33). 


\section{Part II: Solution of the WZNW model}

After all the representation theoretic preparations we can now address the WZNW model on the supergroup PSU $(1,1 \mid 2)$. We shall start with a discussion of the Lagrangian before we proceed to the particle limit and analyze its state space in some detail. We shall show, in particular, that the Laplacian on the supergroup $\operatorname{PSU}(1,1 \mid 2)$ is non-diagonalizable and obtain explicit formulas for all its generalized eigenfunctions. Afterwards we turn to the full field theory. The latter will be solved through a free field representation. We discuss how the non-diagonalizability of the Laplacian is naturally inherited by the zero-mode of the energy momentum tensor. Hence, the PSU $(1,1 \mid 2)$ WZNW model provides an example of a logarithmic conformal field theory. As an application of our results on the structure of the state space, we finally propose an algorithm that allows to count the number of states that possess the same transformation law under the global symmetries. The resulting formulas will only be used in our forthcoming analysis of the RR deformation. Nevertheless, some comments on the latter are included at the end of this part.

\section{The WZNW Lagrangian}

Before we spell out the WZNW model we are about to consider, we would like to recall a few basic facts on supergroups. Let us begin with the supergroup GL $(m \mid n)$. Elements $S \in \mathrm{GL}(m \mid n)$ can be represented through invertible matrices of the form

$$
S=\left(\begin{array}{ll}
A & \sigma \\
\rho & B
\end{array}\right)
$$

where $A$ and $B$ are elements of $\mathrm{GL}(m)$ and $\mathrm{GL}(n)$, respectively, with Grassmann-even matrix elements. The symbols $\sigma$ and $\rho$, on the other hand, denote rectangular matrices with Grassmann-odd entries. We pass from $\operatorname{GL}(m \mid n)$ to $\operatorname{SL}(m \mid n)$ by imposing the additional condition $\operatorname{sdet}(S)=1$ on the superdeterminant of $S$,

$$
\operatorname{sdet}(S)=\frac{\operatorname{det}\left(A-\sigma B^{-1} \rho\right)}{\operatorname{det}(B)}=\frac{\operatorname{det}(A)}{\operatorname{det}\left(B-\rho A^{-1} \sigma\right)} \stackrel{!}{=} 1 \text {. }
$$

When $n=m$, the construction of the superdeterminant implies that $\mathrm{SL}(\mathrm{n} \mid \mathrm{n})$ possesses a non-trivial center consisting of scalar multiples of the identity matrix. In descending to 
$\operatorname{PSL}(n \mid n)$, we identify supermatrices in $\operatorname{SL}(n \mid n)$ that differ by a scalar multiple. Furthermore, we introduce the following unitarity condition for supermatrices $S \in \mathrm{GL}(m \mid n)$,

$$
S \eta S^{\dagger}=\eta
$$

where $\eta=\operatorname{diag}(-1,1, \ldots, 1)$ is the $m+n$ dimensional Minkowski metric. Supermatrices $S \in \mathrm{SL}(m \mid n)$ obeying the condition (4.3) form the supergroup $\mathrm{SU}(1, m-1 \mid n)$. Identification of scalar multiples finally leads to $\operatorname{PSU}(1, m-1 \mid n)$.

We are now prepared to spell out the action functional for the WZNW model on $\operatorname{PSU}(1,1 \mid 2)$ at level $k$. Since there is no fundamental representation of this group we will instead work with $\mathrm{SU}(1,1 \mid 2)$ and show that the Lagrangian actually does not depend on the additional degree of freedom corresponding to multiples of the identity element. For all $S \in \mathrm{SU}(1,1 \mid 2)$ we thus define

$$
\mathcal{S}_{k}^{\mathrm{SU}(1,1 \mid 2)}[S]=-\frac{k}{2 \pi} \int d^{2} z \operatorname{str}\left(S^{-1} \partial S S^{-1} \bar{\partial} S\right)-\frac{k}{12 \pi} \int \operatorname{str}\left(\left(S^{-1} d S\right)^{3}\right)
$$

with a suitably normalized supertrace str. For supermatrices $S$ of the form (4.1), the supertrace is given by $\operatorname{str}(S)=\operatorname{tr}(A)-\operatorname{tr}(D)$. Using the Polyakov-Wiegmann identity for WZNW models,

$$
\mathcal{S}_{k}^{\mathrm{SU}(1,1 \mid 2)}\left[S_{1} S_{2}\right]=\mathcal{S}_{k}^{\mathrm{SU}(1,1 \mid 2)}\left[S_{1}\right]+\mathcal{S}_{k}^{\mathrm{SU}(1,1 \mid 2)}\left[S_{2}\right]+\frac{k}{2 \pi} \int d^{2} z \operatorname{str}\left(S_{1}^{-1} \bar{\partial} S_{1} \partial S_{2} S_{2}^{-1}\right),
$$

one may easily show that the action $\mathcal{S}_{k}^{\mathrm{SU}(1,1 \mid 2)}$ remains invariant if we multiply the supermatrix $S \in \mathrm{SU}(1,1 \mid 2)$ with a scalar factor $\exp \Phi$, i.e.

$$
\mathcal{S}_{k}^{\mathrm{SU}(1,1 \mid 2)}\left[e^{\Phi} S\right]=\mathcal{S}_{k}^{\mathrm{SU}(1,1 \mid 2)}[S] .
$$

This relation ensures that the functional form of the WZNW action for PSU $(1,1 \mid 2)$ is identical to the WZNW action for $\mathrm{SU}(1,1 \mid 2)$. In particular we like to stress that no explicit gauge procedure is required, in contrast to what has been proposed in [9]. As in all WZNW models the Lagrangian defined in (4.4) leads to two chiral sets of currents $J^{\mu}(z)$ and $\bar{J}^{\mu}(\bar{z})$ which generate two (anti)commuting copies of the affine Lie superalgebra $\widehat{\operatorname{psu}}(1,1 \mid 2)_{k}$. Their precise relations can easily be reconstructed from its zero-mode subsuperalgebra (2.1) and the metric (2.2).

Our aim is to show that the introduction of auxiliary fields allows to decouple bosonic and fermionic degrees of freedom to a large extent. The result will be a sum of two renormalized bosonic WZNW models, the action for a holomorphic and anti-holomorphic 
set of symplectic fermions and an interaction term which mixes the two. Our derivation is motivated by the ideas given in [9] but the fermions are treated differently, along the lines of [20]. The first step is to rewrite our action with the help of the following product decomposition of $\mathrm{SU}(1,1 \mid 2)$ supermatrices,

$$
S=e^{\Phi}\left(\begin{array}{cc}
\text { id } & 0 \\
\lambda & \text { id }
\end{array}\right) \cdot\left(\begin{array}{cc}
A & 0 \\
0 & B
\end{array}\right) \cdot\left(\begin{array}{cc}
\text { id } & \chi \\
0 & \text { id }
\end{array}\right)=e^{\Phi}\left(\begin{array}{cc}
A & A \chi \\
\lambda A & \lambda A \chi+B
\end{array}\right)
$$

where the factor $\exp \Phi$ is chosen such that the matrices $A$ and $B$ are uni-modular. Using once more the Polyakov-Wiegmann identity (4.5) for supergroups, we find that

$$
\begin{aligned}
& \mathcal{S}_{k}^{\mathrm{PSU}(1,1 \mid 2)}[S]=\mathcal{S}_{k}^{\mathrm{PSU}(1,1 \mid 2)}\left[\left(\begin{array}{cc}
\mathrm{id} & 0 \\
\lambda & \mathrm{id}
\end{array}\right)\right]+\mathcal{S}_{k}^{\mathrm{PSU}(1,1 \mid 2)}\left[\left(\begin{array}{cc}
A & 0 \\
0 & B
\end{array}\right)\right]+\mathcal{S}_{k}^{\mathrm{PSU}(1,1 \mid 2)}\left[\left(\begin{array}{cc}
\mathrm{id} & \chi \\
0 & \text { id }
\end{array}\right)\right] \\
& +\frac{k}{2 \pi} \int d^{2} z \operatorname{str}\left(\begin{array}{cc}
0 & 0 \\
\bar{\partial} \lambda \partial A A^{-1} & \bar{\partial} \lambda A \partial \chi B^{-1}
\end{array}\right)
\end{aligned}
$$

The first and third term vanishes due to the fact that the only contributions to supertraces come from non-trivial bosonic submatrices. Hence, we are left with only two terms. Taking into account the indefinite structure of the metric the result can now we rewritten as

$$
\mathcal{S}_{k}^{\mathrm{PSU}(1,1 \mid 2)}[S]=\mathcal{S}_{k}^{\mathrm{AdS}_{3}}[A]+\mathcal{S}_{k}^{\mathrm{SU}(2)}[B]-\frac{k}{2 \pi} \int d^{2} z \operatorname{tr}\left[\bar{\partial} \lambda A \partial \chi B^{-1}\right]
$$

where $\mathcal{S}_{k}^{\mathrm{AdS}_{3}}$ and $\mathcal{S}_{k}^{\mathrm{SU}(2)}$ denote the usual bosonic WZNW actions. It is easy to see that the previous action is equivalent to the functional

$$
\mathcal{S}_{k}^{\mathrm{PSU}(1,1 \mid 2)}[S, p, \theta]=\mathcal{S}_{k+2}^{\mathrm{AdS}_{3}}[A]+\mathcal{S}_{k-2}^{\mathrm{SU}(2)}[B]+\frac{1}{2 \pi k} \int d^{2} z \operatorname{tr}\left\{k(p \bar{\partial} \theta+\bar{p} \partial \bar{\theta})+A^{-1} p B \bar{p}\right\}
$$

where the fields have been decoupled to a large extent. Integrating out the auxiliary fields $p$ and $\bar{p}$ one indeed arrives at the original action if one imposes the identifications $\theta=\lambda$ and $\bar{\theta}=\chi$. The shift of the levels arises from the modification of the path integral measure.

\section{The minisuperspace theory}

In this section we would like to analyse the space of (generalized) eigenfunctions of the Laplace operator on the supergroup $\operatorname{PSU}(1,1 \mid 2)$. We shall set up the problem in the first subsection. Explicit formulas for all the generalized eigenfunctions are derived in the second subsection. Their transformation behaviour with respect to the left and right regular action of $\operatorname{psl}(2 \mid 2)$ is finally investigated in the last subsection. 


\subsection{The Laplacian on the Supergroup PSU $(1,1 \mid 2)$}

On the supergroup $\operatorname{PSU}(1,1 \mid 2)$ we can introduce various different coordinates. For our purposes in the next subsection, a preferred set of coordinates is defined through the decomposition,

$$
G=e^{i \eta_{a} S_{2}^{a}} g e^{i \bar{\eta}_{a} S_{1}^{a}}=e^{i \eta_{a} S_{2}^{a}} e^{i x_{a b} K^{a b}} e^{i \bar{\eta}_{a} S_{1}^{a}}
$$

In these coordinates, we can easily express the differential operators implementing the left and right regular representation. In the following, we denote the generators of the bosonic subalgebra on the bosonic subgroup by $L_{0}^{a b}$ and $R_{0}^{a b}$, respectively. For the left regular action we find (see also [39])

$$
\begin{gathered}
L_{2}^{a}=\partial^{a} \quad, \quad L^{a b}=L_{0}^{a b}-i\left(\eta^{a} \partial^{b}-\eta^{b} \partial^{a}\right) \\
L_{1}^{a}=-D^{a b}(g) \bar{\partial}^{b}+\tilde{L}_{1}^{a} \quad \text { where } \tilde{L}_{1}^{a}=\frac{i}{2} \epsilon^{a b c d}\left(\eta^{b} L_{0}^{c d}-i \eta^{b} \eta^{c} \partial^{d}\right)
\end{gathered}
$$

Here, the partial derivatives $\partial^{a}$ and $\bar{\partial}^{a}$ denote differentiation with respect to the fermionic coordinates $\eta_{a}$ and $\bar{\eta}_{a}$, respectively, and the matrix $D^{a b}(g)$ of functions on the bosonic subgroup is obtained by evaluation of elements $g$ in the $(1 / 2,1 / 2)$ representation. By construction, it satisfies the following differential equations

$$
L_{0}^{a b} D^{c d}(g)=i\left(\delta^{a c} D^{b d}(g)-\delta^{b c} D^{a d}(g)\right), \quad R_{0}^{a b} D^{c d}(g)=i\left(\delta^{a d} D^{c b}(g)-\delta^{b d} D^{c a}(g)\right)
$$

To check the commutation relations is straightforward, though a bit cumbersome. The right regular representation is obtained similarly, with the two types of fermionic generators exchanged, $\bar{\eta}_{a}$ replacing $\eta_{a}$ etc. Needless to stress that the left and right action (anti-)commute.

A short and straightforward computation of the quadratic Casimir element $C_{2}=$

$-\epsilon_{a b c d} K^{a b} K^{c d} / 4+\epsilon_{\alpha \beta} S_{\alpha}^{a} S_{\beta}^{a}$ in the left or right regular representation gives the following explicit formula for the Laplacian $\Delta$ on the supergroup

$$
\Delta=L\left(C_{2}\right)=R\left(C_{2}\right)=\Delta_{0}+Q \quad \text { where } \quad Q=2 \partial_{a} D^{a b}(g) \bar{\partial}_{b}
$$

and $\Delta_{0}$ is the usual Laplace operator on the bosonic subgroup, i.e. on $\operatorname{AdS}_{3} \times \mathrm{S}^{3}$. Our aim is to find generalized eigenfunctions of this operator. Let us recall that a function $\psi$ is called a generalized eigenfunction of $\Delta$ for eigenvalue $\lambda$ if

$$
(\Delta-\lambda)^{n} \psi=0 \quad \text { for some } \quad n>0
$$


$\psi$ is an eigenfunction if this equation is satisfied for $n=1$ (and hence for all other values of $n$ ). We shall see in the next subsection that generalized eigenfunctions of $\Delta$ with $\lambda \neq 0$ are in fact true eigenfunctions. For $\lambda=0$, on the other hand, non-trivial generalized eigenfunctions do appear. This means that the Laplacian on the supergroup PSU $(1,1 \mid 2)$ can only be brought into Jordan normal form. The Jordan cells turn out to possess a rank up to five. By construction, the individual spaces of generalized eigenfunctions come equipped with the left and right regular action of the Lie superalgebra psl $(2 \mid 2)$. We shall describe its decomposition into indecomposables in the second subsection.

\subsection{Generalized eigenfunctions of the Laplacian}

In this subsection we show that generalized eigenfunctions of the Laplacian on $\operatorname{PSU}(1,1 \mid 2)$ are in one-to-one correspondence with elements of the following auxiliary space

$$
\mathcal{H}_{0}:=L_{2}\left(\mathrm{AdS}_{3} \times \mathrm{SU}(2)\right) \otimes \Lambda(\eta, \bar{\eta})
$$

of Grassmann valued functions whose coefficients are square integrable functions on the bosonic subgroup. Under favorable circumstances, i.e. when the fermionic generators of the Lie superalgebra transform in a unitary representation of its bosonic subgroup $G$, the space of Grassmann valued functions on $G$ coincides with the space of generalized eigenfunctions. This is the case e.g. for the GL(1|1) model studied in [20], but the key example we have in mind here is $\operatorname{PSL}(2 \mid 2)$ with a real form that removes all non-trivial finite dimensional representations of the bosonic subalgebra, and in particular the $(1 / 2,1 / 2)$ representation, from the list of unitaries.

The key idea in the subsequent construction of generalized eigenfunctions is to consider the elements $\psi_{0}$ of $\mathcal{H}_{0}$ as 'leading contributions'. More precisely, we shall show that all eigenfunctions of the bosonic Laplacian can be turned into generalized eigenfunctions by adding appropriate terms with lower fermion number. To this end, let us rewrite equation (5.5) in the following form

$$
(\Delta-\lambda)^{n} \psi_{\lambda}=\left(\left(\Delta_{0}-\lambda\right)^{n}+A_{n}(\lambda)\right) \psi_{\lambda}=0
$$

The operators $A_{n}(\lambda)$ are lengthy combinations of $Q$ and $\left(\Delta_{0}-\lambda\right)$ which can be worked out explicitly. What is most important is to note that each term in $A_{n}(\lambda)$ contains a least one $Q$. Hence, the operators $A_{n}(\lambda)$ are nilpotent. 
A short and formal manipulation shows that generalized eigenfunctions for the eigenvalue $\lambda$ at order $n$ possess the general form

$$
\psi_{\lambda}^{(n)}=\Xi_{\lambda}^{(n)} \psi_{0}^{\lambda}=\sum_{\nu=0}^{\infty}\left(-\left(\Delta_{0}-\lambda\right)^{-n} A_{n}(\lambda)\right)^{\nu} \psi_{0}^{\lambda}
$$

where $\psi_{0}^{\lambda} \in \mathcal{H}_{0}$ is an eigenfunction of $\Delta_{0}$ with eigenvalue $\lambda$, i.e. $\Delta_{0} \psi_{0}^{\lambda}=\lambda \psi_{0}^{\lambda}$. Since $A_{n}(\lambda)$ contains anti-commuting fermionic derivatives, the sum on the right hand side truncates after a finite number of terms at $\nu=4$. On the other hand, the formula requires to invert the operator $\Delta_{0}-\lambda$. Hence the operator $\Xi_{\lambda}^{(n)}$ need not be well defined for all $\psi_{0}^{\lambda}$. To analyze this issue further, we note that $\Xi_{\lambda}^{(n)}=\Xi_{\lambda}^{(5)}=\Xi_{\lambda}$ is independent of $n$ for $n \geq 5$ and compute

$$
\begin{gathered}
\psi_{\lambda}=\Xi_{\lambda} \psi_{0}^{\lambda}=\psi_{0}^{\lambda}-\frac{1}{\left(\Delta_{0}-\lambda\right)} Q_{\lambda}^{\prime} \psi_{0}^{\lambda}+\left(\frac{1}{\left(\Delta_{0}-\lambda\right)} Q_{\lambda}^{\prime}\right)^{2} \psi_{0}^{\lambda}+\frac{1}{\left(\Delta_{0}-\lambda\right)^{2}} Q_{\lambda}^{\prime} Q_{\lambda}^{\prime \prime} \psi_{0}^{\lambda}+\ldots \\
\text { where } Q=Q_{\lambda}^{\prime}+Q_{\lambda}^{\prime \prime}=\left(1-P_{0}(\lambda)\right) Q+P_{0}(\lambda) Q
\end{gathered}
$$

and $P_{0}(\lambda)$ is the projector on eigenstates of $\Delta_{0}$ with eigenvalue $\lambda$. We have not displayed the third and fourth order terms in $Q$ because the expression would be rather bulky (there are 14 such terms). Let us observe that the inverse powers of $\left(\Delta_{0}-\lambda\right)$ only act in combination with $Q_{\lambda}^{\prime}$, i.e. after application of the projection $1-P_{0}(\lambda)$. This continues to hold for the higher order terms and hence $\psi_{\lambda}$ is well defined for all $\psi_{0}^{\lambda}$. Since $P_{0}(\lambda) \Xi_{\lambda}=\mathrm{id}$, we conclude that generalized eigenfunctions are indeed in one-to-one correspondence with elements $\psi_{0} \in \mathcal{H}_{0}$.

It is instructive to contrast these findings with results on the true eigenvectors of the Laplacian. Our general formula (5.5) applied to the special case $n=1$ shows that such eigenfunctions must be of the form

$$
\begin{aligned}
\psi_{\lambda}^{(1)} & =\Xi_{\lambda}^{(1)} \psi_{0}^{\lambda}=\sum_{\nu=0}^{\infty}\left(-\left(\Delta_{0}-\lambda\right)^{-1} Q\right)^{\nu} \psi_{0}^{\lambda} \\
& =\psi_{0}^{\lambda}-\frac{1}{\left(\Delta_{0}-\lambda\right)} Q \psi_{0}^{\lambda}+\left(\frac{1}{\left(\Delta_{0}-\lambda\right)} Q\right)^{2} \psi_{0}^{\lambda}+\ldots
\end{aligned}
$$

In order for $\psi_{\lambda}^{(1)}$ to be well defined it is obviously necessary that $Q_{\lambda}^{\prime \prime} \psi_{0}^{\lambda}=P_{0}(\lambda) Q \psi_{0}^{\lambda}=0.11$ Note that the condition automatically ensures that our expression for the eigenfunction

\footnotetext{
${ }^{11}$ The condition is also sufficient, though this requires a slightly more elaborate argument.
} 
$\psi_{\lambda}^{(1)}$ agrees with the formula for generalized eigenfunctions above. It is easy to see that

$$
P_{0}(\lambda) Q \psi_{0}^{\lambda}=0 \quad \text { for eigenfunctions } \psi_{0}^{\lambda} \text { of } \Delta_{0} \text { with eigenvalue } \lambda \neq 0
$$

Hence, we conclude that all generalized eigenfunctions of the Laplacian on PSU $(1,1 \mid 2)$ with nonzero eigenvalue are true eigenfunctions. For $\lambda=0$, on the other hand, non-trivial generalized eigenfunctions exist. These are in one-to-one correspondence with functions $\psi_{0}^{\lambda}$ for which $P_{0}(0) Q \psi_{0}^{\lambda} \neq 0$.

Our claim (5.9) can be established as follows: suppose that $\psi_{0}^{\lambda}$ transforms in the representation $\left(j_{1}, j_{2}\right)$ of the bosonic subalgebra. Then, after application of $Q$, the resulting state $Q \psi_{0}^{\lambda}$ decomposes into four composents according to the four different representations $\left(j_{1} \pm 1 / 2, j_{2} \pm 1 / 2\right)$ and $\left(j_{1} \pm 1 / 2, j_{2} \mp 1 / 2\right)$ that arise after multiplication with the functions $D^{a b}(g)$ in the $(1 / 2,1 / 2)$ representations. The resulting possible eigenvalues of the bosonic Laplacian are $\delta_{ \pm}=\lambda \pm\left(j_{1}-j_{2}\right)$ and $\delta_{ \pm}=\lambda \pm\left(j_{1}+j_{2}-1\right)$ with $\lambda=j_{1}\left(j_{1}+1\right)-j_{2}\left(j_{2}+1\right)$. Hence, we conclude that $P_{0}(\lambda) Q \psi_{0}^{\lambda}=0$, unless $j_{1}=j_{2}$ or $j_{1}+1=-j_{2}$. The latter conditions on the choice of $\left(j_{1}, j_{2}\right)$ are equivalent to requiring $\lambda=0$. This proves our claim and concludes this subsection.

\subsection{Regular action on generalized eigenfunctions}

Since the Laplacian commutes with both the left and the right regular representation, $L$ and $R$ provide two (anti-)commuting actions of the Lie superalgebra psl(2|2) on generalized eigenfunctions $\psi \in \mathcal{H} \equiv \Xi \mathcal{H}_{0}$.

In order to spell out the behavior of states $\psi \in \mathcal{H}$ under the right regular action we introduce the following new representations

$$
B(\mu, \nu):=\operatorname{Ind}_{\mathfrak{g}^{(0)}}^{\mathfrak{g}} V_{(\mu, \nu)}=\mathcal{U}(\mathfrak{g}) \otimes_{\mathfrak{g}^{(0)}} V_{(\mu, \nu)}
$$

By construction, these representations have a dimension $16^{2} \cdot \operatorname{dim}(\mu, \nu)$ and certainly none of them is irreducible. All the representations can be decomposed into a sum of projective representations [29]. Generically, $B(\mu, \nu)$ decomposes into a sum of typical Kac modules according to

$$
B(\mu, \nu) \cong \bigoplus_{\left(\mu^{\prime}, \nu^{\prime}\right)}\left[[\mu, \nu]:\left(\mu^{\prime}, \nu^{\prime}\right)\right] \cdot\left[\mu^{\prime}, \nu^{\prime}\right]
$$

where $\left[[\mu, \nu]:\left(\mu^{\prime}, \nu^{\prime}\right)\right]$ denotes the multiplicity of the bosonic multiplet $\left(\mu^{\prime}, \nu^{\prime}\right)$ inside the Kac module $[\mu, \nu]$. The formula applies whenever the summation extends only over 
bosonic representations $\left(\mu^{\prime}, \nu^{\prime}\right)$ with non-vanishing Casimir. More explicitly, we can use the above formula for all representations $(\mu, \nu)=\left(\left(j_{1}, \alpha\right), j_{2}\right)$ from the continuous series and for discrete series representations $(\mu, \nu)=\left(\left( \pm, j_{1}\right), j_{2}\right)$ as long as $j_{1}+j_{2}+1 \neq 0, \pm 1$. In the remaining cases, projective covers of atypical representations appear,

$$
\begin{aligned}
B(( \pm,-j-1), j) & \cong 2 \cdot \mathcal{P}_{j}^{ \pm} \oplus \ldots \\
B\left(\left( \pm,-j-\frac{1}{2}\right), j+\frac{1}{2}\right) & \cong \mathcal{P}_{j-\frac{1}{2}}^{ \pm} \oplus \ldots \\
B\left(\left( \pm,-j-\frac{3}{2}\right), j-\frac{1}{2}\right) & \cong \mathcal{P}_{j+\frac{1}{2}}^{ \pm} \oplus \ldots
\end{aligned}
$$

where the dots ... stand for a sum of typical Kac modules that can be determined through the rule (5.10) if we remember to omit all terms that correspond to an atypical representation.

It is relatively easy to see that the space $\mathcal{H}$ of generalized eigenfunctions possesses the following decomposition with respect to the asymmetric action of the subsymmetry $\mathfrak{g}_{L}^{(0)} \oplus \mathfrak{g}_{R}$,

$$
\begin{aligned}
\mathcal{H} \cong \bigoplus_{2 J=0}^{\infty} \int_{\mathbb{S}} d j \int_{0}^{1} d \alpha((j, \alpha), J)_{L}^{+} \otimes B((j, \alpha), J)_{R} \oplus \\
\oplus \bigoplus_{2 J=0}^{\infty} \bigoplus_{\eta= \pm} \int_{\frac{1}{2}}^{\infty} d j((\eta,-j), J)_{L}^{+} \otimes B((\eta,-j), J)_{R}
\end{aligned}
$$

The domain of the first integral is given by $\mathbb{S}=-1 / 2+i \mathbb{R}$, as usual for the principal continuous series representations. In order to justify the decomposition, let us note that the ground states from which the representations $B(\mu, \nu)$ are generated, can be identified with those states $\psi_{\lambda}$ in $\mathcal{H}$ whose top component $P_{0}(\lambda) \psi_{\lambda}$ has maximal fermion number.

In order to rewrite our decomposition formula in terms of indecomposables, we need to insert the formulas (5.10)-(5.13). We then collect all terms that give rise to the same projective representation of $\mathfrak{g}_{R}$. On the subspace $\mathcal{H}_{\text {typ }} \subset \mathcal{H}$ of states with non-zero eigenvalue $\lambda$, the bosonic multiplets of of the $\mathfrak{g}_{L}^{(0)}$ action turn out to combine into a Kac module for the Lie superalgebra $\mathfrak{g}_{L}$, i.e.

$$
\begin{aligned}
\mathcal{H}_{\mathrm{typ}} \cong \bigoplus_{2 J=0}^{\infty} \int_{\mathbb{S}} d j \int_{0}^{1} d \alpha[(j, \alpha), J]_{L}^{+} \otimes[(j, \alpha), J]_{R} \oplus \\
\oplus \bigoplus_{2 J=0}^{\infty} \bigoplus_{\eta= \pm} \int_{\frac{1}{2}, j \neq J+1}^{\infty} d j[(\eta,-j), J]_{L}^{+} \otimes[(\eta,-j), J]_{R}
\end{aligned}
$$


Another way to arrive at this result is by noting that our operator $\Xi$ provides an intertwiner between the action of $L$ and $R$ on $\mathcal{H}_{\text {typ }}$ and some simplified action $\tilde{L}$ and $\tilde{R}$ of $\operatorname{psl}(2 \mid 2)$ on the subspace $\mathcal{H}_{0, \text { typ }} \subset \mathcal{H}_{0}$ of eigenfunctions with non-zero eigenvalues of $\Delta_{0}$. The action $\tilde{L}$, for example, is generated by the operator $\tilde{L}_{1}^{a}$ defined in formula (5.3) along with

$$
\tilde{L}_{2}^{a}=L_{2}^{a} \quad, \quad \tilde{L}^{a b}=L^{a b} .
$$

A similar construction gives $\tilde{R}$. With respect to these two actions of $\operatorname{psl}(2 \mid 2)$, the space $\mathcal{H}_{0}$ is easily seen to decompose into an integral over left and right Kac modules. This applies even to the subspace on which $\Delta_{0}$ vanishes. But on the latter $\Xi$ ceases to be an intertwiner between the truncated actions $\tilde{L}, \tilde{R}$ and the full regular action $L, R$. In the next section we shall see that the $\tilde{L}-\tilde{R}$ module $\mathcal{H}_{0}$ models the space of vertex operators in the free field representation. The full $L-R$ action on $\mathcal{H}$, on the other hand, agrees with actions of $\operatorname{psl}(2 \mid 2)$ on the ground states of the full interacting theory. The discrepancy between the two actions in the atypical sector will have remarkable consequences which at the end culminate in the logarithmic behavior of the WZNW theory.

As for the atypical sector $\mathcal{H}_{\text {atyp }}$ of generalized eigenfunctions with vanishing eigenvalue $\lambda$, it is built up from projective covers only when considered with respect to the right (or left) regular action. The associated multiplicity spaces possess the same $\mathfrak{g}_{L}^{(0)}$ representation content as the atypical irreducible $\operatorname{psl}(2 \mid 2)$ representations from the discrete series. But this time, enhancing the left action from the bosonic subalgebra to the full $\operatorname{psl}(2 \mid 2)$ has more drastic effects than simply to promote the multiplicity spaces into representations of the Lie superalgebra. Note that such a behavior would obviously violate the symmetry between left and right regular transformations and hence cannot be the right answer. Instead, as a $\mathfrak{g}_{L} \oplus \mathfrak{g}_{R}$ module, the atypical sector $\mathcal{H}$ is built from non-chiral indecomposables which encompass an infinite number of atypical constituents much in the same way as it happens for GL(1|1) (see [20]). We refrain from working out the details here.

This gives us a fairly complete picture of the space of wave functions for a particle moving on PSU $(1,1 \mid 2)$ and a very good basis to discuss how field theoretic corrections affect the structure of the state space. In the full field theory, there will be two new phenomena which have to be taken into account. First of all there will be a cut-off associated with the finiteness of the level $k$. Moreover, the affine Lie superalgebra admits a family of spectral flow automorphisms which has to be taken into account properly. 


\section{Vertex operators and correlation functions}

Now that we obtained a profound knowledge about the particle limit of the sigma model on $\operatorname{PSU}(1,1 \mid 2)$, we are finally in a position to return to the solution of the full quantum theory. Our starting point is the Lagrangian (4.9),

$$
\mathcal{S}_{k}^{\mathrm{PSU}(1,1 \mid 2)}=\mathcal{S}_{0}+\mathcal{S}_{\mathrm{int}}=\mathcal{S}_{k+2}^{\mathrm{AdS}}+\mathcal{S}_{k-2}^{\mathrm{SU}(2)}+\mathcal{S}_{\mathrm{ferm}}+\mathcal{S}_{\mathrm{int}},
$$

consisting of a decoupled system with a purely bosonic WZNW model on $\mathrm{AdS}_{3} \times \mathrm{SU}(2)$ and a set of free fermions as well as an interaction term coupling bosonic and fermionic degrees of freedom. Following the general strategy adopted in [20] we will start with an analysis of the decoupled theory and consider the additional term as a perturbation. Our main aim is to find the vertex operators of the full supergroup WZNW theory and to sketch the calculation of their correlation functions.

The state space $\hat{\mathcal{H}}_{0}$ of the decoupled theory described by $\mathcal{S}_{0}$ is completely known using standard results in conformal field theory. For reasons to become clear below we restrict ourselves to fermions with integer moding. Under this assumption there exists a unique representation $\mathcal{F} \otimes \overline{\mathcal{F}}$ for the fermions. It is generated from a ground state by the application of the modes $\theta_{-n}^{a}$ and $p_{-(n+1)}^{a}$ for $n \geq 0$ and similarly for the antiholomorphic fields. The $\mathrm{SU}(2)_{k-2}$ WZNW model is described by a charge conjugate partition function involving unitary representations with spin $2 J=0,1, \ldots, k-2$ [40]. In the $\mathrm{AdS}_{3}$ WZNW model on the other hand two different kinds of representations of the underlying affine algebra $\widehat{\mathrm{sl}}(2, \mathbb{R})_{k+2}$ contribute [13]: the principal continuous series $(j, \alpha)$ for $j \in \mathbb{S}=-\frac{1}{2}+i \mathbb{R}$ and $\alpha \in[0,1)$ and the discrete series $( \pm, j)$ for $-\frac{1}{2}>j>-\frac{k+1}{2}$. Moreover, one has to take into account the spectral flow automorphism which maps ordinary highest weight modules to twisted ones. This leads to an additional quantum number $w$ which has to be attached to the representations of $\widehat{\operatorname{sl}}(2, \mathbb{R})_{k+2}$. The precise definition of the spectral flow automorphism has been given in (3.17).

Based on the previous remarks we can spell out the space of the decoupled system, 12

$$
\begin{aligned}
\hat{\mathcal{H}}_{0} \cong \bigoplus_{2 J=0}^{k-2} \bigoplus_{w \in \mathbb{Z}} \int_{\mathbb{S}} d j \int_{0}^{1} d \alpha\left[\mathcal{V}_{\left((j, \alpha)_{w}, J\right)} \otimes \mathcal{F}\right] \otimes\left[\mathcal{V}_{\left((j, \alpha)_{w}, J\right)^{+}} \otimes \overline{\mathcal{F}}\right] \\
\oplus \bigoplus_{2 J=0}^{k-2} \bigoplus_{w \in \mathbb{Z}} \int_{\frac{1}{2}}^{\frac{k+1}{2}} d j\left[\mathcal{V}_{\left((+,-j)_{w}, J\right)} \otimes \mathcal{F}\right] \otimes\left[\mathcal{V}_{\left((+,-j)_{w}, J\right)^{+}} \otimes \overline{\mathcal{F}}\right] .
\end{aligned}
$$

\footnotetext{
${ }^{12}$ We remind the reader that the orbit of representations $(+, j)_{w}$ includes representations based on $\left(-, j^{\prime}\right)$.
} 
Nevertheless we are not yet done. Since we intend to describe a supersymmetric theory we have to cast the state space in a manifestly covariant form. In addition we have to make contact to the minisuperspace analysis presented in section 5. In order to achieve these goals we must find a realization of each, the holomorphic and anti-holomorphic affine Lie superalgebras $\widehat{\operatorname{psu}}(1,1 \mid 2)_{k}$, on $\hat{\mathcal{H}}_{0}$. Moreover their zero mode action on the ground states has to resemble that of the differential operators $\tilde{L}$ and $\tilde{R}$ on $\mathcal{H}_{0}$, respectively. This space and the corresponding operators have been introduced in (5.6) and (5.14).

In fact, a realization of the affine Lie superalgebra $\widehat{\operatorname{psu}}(1,1 \mid 2)_{k}$ in terms of the symmetry generators $j^{a b}$ and the fermions $p$ and $\theta$ of the decoupled system (and their antiholomorphic analogues) has already been presented in (3.16). In this case the zero mode sector of the corresponding expressions indeed reduces to the tilded differential operators (5.14) acting on the space $\mathcal{H}_{0}$ if we identify the auxiliary fields $p$ and $\bar{p}$ with the fermionic derivatives as usual. For the identification to hold it is crucial that in the first term $\partial \theta^{a}$ in $S_{1}^{a}$ the zero mode of the coordinate field $\theta$ is eliminated by the action of the derivative. Needless to say, similar considerations apply for the anti-holomorphic sector.

After having established the structure of $\hat{\mathcal{H}}_{0}$ as a representation space with respect to $\widehat{\operatorname{psu}}(1,1 \mid 2)_{k} \oplus \widehat{\operatorname{psu}}(1,1 \mid 2)_{k}$ it is just a small step to spell out the proposal

$$
\hat{\mathcal{H}}_{0} \cong \bigoplus_{2 J=0}^{k-2} \bigoplus_{w \in \mathbb{Z}} \int_{\mathbb{S}} d j \int_{0}^{1} d \alpha \mathcal{V}_{[(j, \alpha), J]_{w}} \otimes \mathcal{V}_{[(j, \alpha), J]_{w}^{+}} \oplus \bigoplus_{2 J=0}^{k-2} \bigoplus_{w \in \mathbb{Z}} \int_{\frac{1}{2}}^{\frac{k+1}{2}} d j \mathcal{V}_{[(+,-j), J]_{w}} \otimes \mathcal{V}_{[(+,-j), J]_{w}^{+}}
$$

This space meets all the requirements stated above. First of all, it is indeed a fully covariant version of the space (6.2). This is immediately obvious in view of our discussion of affine Lie superalgebra representations in section 3.2 and, in particular, given the definition (3.24). Only the treatment of spectral flow requires a few comments since a spectral flow which exclusively acts in the $\mathrm{AdS}_{3}$ sector cannot be lifted to the full superalgebra $\operatorname{PSU}(1,1 \mid 2)$ in general. Indeed, as can be inferred from (3.17) the only spectral flow automorphisms which solely act on the AdS factor are of the form $\gamma^{(w, 0)}$. But in order to keep the integer moding of the fermions which is required for the implementation of the global supersymmetry one would have to choose $w$ even, resulting in the ommission of every second representation. The simple way out is to consider the spectral flows $\gamma^{(w, w)}$ for all $w \in \mathbb{Z}$. In this case the moding of the fermions stays invariant and the action on the $\mathrm{SU}(2)$ sector can be absorbed in a relabeling of the corresponding integrable weights. Spectral flow automorphisms that respect the fermionic boundary conditions were found 
in [20] to be exact symmetries of the WZNW model on the supergroup GL(1|1). We believe that this observation generalizes to arbitrary supergroups. In the case of $\operatorname{PSU}(1,1 \mid 2)$ it is indeed consistent with the results of Maldacena and Ooguri [13].

On the other hand, beside being supersymmetric, the state space (6.3) can be shown to be a straightforward affinization of the minisuperspace result $\mathcal{H}_{0}$. In order to establish this correspondence we consider the semi-classical limit $k \rightarrow \infty$ in which the curvature of the supergroup becomes small and the truncation of the spectrum can be neglected. We are moreover only interested in the light states whose conformal dimension approach zero. This forces us to discard all the spectral flow representations 13 We are thus left with the ground states of the affine modules $\mathcal{V}_{[\mu, \nu]}$ and these obviously transform in the Kac module $V_{[\mu, \nu]}$. This concludes our treatment of the decoupled theory.

Now we turn our attention again to the full WZNW model as defined in eq. (6.1), including the interaction term $\mathcal{S}_{\text {int }}$. Let us remind the reader that the space $\mathcal{H}_{0}$ just has been an auxiliary space which helped analyzing the space $\mathcal{H}=\Xi \mathcal{H}_{0}$ on which the true left and right regular actions of $\operatorname{psu}(1,1 \mid 2)$ have been defined. The same happens in the full $\operatorname{PSU}(1,1 \mid 2)$ field theory where the regular actions are promoted to local symmetries. Roughly speaking, the presence of the additional term $\mathcal{S}_{\text {int }}$ imitates the action of the operator $\Xi: \mathcal{H}_{0} \rightarrow \mathcal{H}$ and modifies the definition of the affine currents. This means that the true state space of the PSU $(1,1 \mid 2)$ WZNW model is given by a space $\hat{\mathcal{H}}$ which differs from $\hat{\mathcal{H}}_{0}$ in the way the affine currents act. Without going into details we symbolically introduce the map $\hat{\Xi}: \hat{\mathcal{H}}_{0} \rightarrow \hat{\mathcal{H}}$ which intertwines the actions in the typical sector. It is important, however, to emphasize that the representation content of $\hat{\mathcal{H}}_{0}$ and $\hat{\mathcal{H}}$ is not isomorphic. In particular, the atypical sector in $\hat{\mathcal{H}}$ may not be written as the product of holomorphic and anti-holomorphic representations since the zero modes $L_{0}$ and $\bar{L}_{0}$ of the energy momentum tensors, the affine analogues of the Laplace operator discussed in section 5, are not diagonalizable. Modular invariance then enforces that the difference of the nilpotent part vanishes on the state space and this is only possible if the representations do not factorize. Another consequence of the previous statements is the occurence of logarithmic correlation functions in the PSU $(1,1 \mid 2)$ WZNW model.

Let us conclude this section with a brief sketch of the calculation of correlation functions. Given any vertex operator $\Phi(z, \bar{z})$ corresponding to a state in the full Hilbert space $\hat{\mathcal{H}}$ - with or without spectral flow - we can find a vertex operator $\Phi_{0}(z, \bar{z})$ in the decou-

\footnotetext{
${ }^{13}$ We should obviously keep those which map $(+, j)$ to $\left(-, j^{\prime}\right)$.
} 
pled theory such that $\Phi(z, \bar{z})=\hat{\Xi} \Phi_{0}(z, \bar{z})$. As in the minisuperspace theory the action of $\hat{\Xi}$ basically adds subleading contributions to the full vertex operator. The correlation functions are then easily determined using the description

$$
\left\langle\Phi\left(z_{1}, \bar{z}_{1}\right) \cdots \Phi\left(z_{n}, \bar{z}_{n}\right)\right\rangle_{\mathrm{PSU}(1,1 \mid 2)_{k}}=\left\langle\Phi_{0}\left(z_{1}, \bar{z}_{1}\right) \cdots \Phi_{0}\left(z_{n}, \bar{z}_{n}\right) e^{-\mathcal{S}_{\mathrm{int}}}\right\rangle_{\mathcal{S}_{0}} .
$$

In order to make sense out of this expression it is necessary to cast the interaction term in a form which may be evaluated in the decoupled theory. It is not difficult to convince oneself that the corresponding alternative form of the interaction term in (4.9) is given by

$$
\mathcal{S}_{\text {int }} \sim \frac{1}{2 \pi k} \int d^{2} z p_{a}(z) D^{a b}(z, \bar{z}) \bar{p}_{b}(\bar{z})
$$

The operators $D^{a b}(z, \bar{z})$ are non-chiral vertex operators of the $\mathrm{AdS}_{3} \times \mathrm{S}^{3}$ WZNW theory which transforms in the $(1 / 2,1 / 2) \times(1 / 2,1 / 2)$ representation with respect to the holomorphic and anti-holomorphic bosonic currents. Given the knowledge of correlation functions in the decoupled theory [41, 14] it is now a tedious but algorithmic exercise to calculate the right hand side of (6.4). It is worth noticing that due to the presence of the fermions $p$ and $\bar{p}$ the expansion will terminate after a finite number of terms. The only caveat concerns the insertion of the vertex operators $D^{a b}$ in the correlation functions of the $\mathrm{AdS}_{3}$ WZNW model since these do not exist in the physical spectrum of the bosonic theory but are rather associated with non-normalizable degenerate fields. It is well known that correlation functions with insertions of such degenerate fields can be determined from the physical ones by analytic continuation. In fact, a reversal of this argument was a crucial ingredient in the solution of the two best understood non-rational conformal field theories, i.e. Liouville theory [42] and the (euclidean) $\mathrm{AdS}_{3}$ model [16]. Hence, all the ingedients for the computation of correlators in the WZNW model on the supergroup are determined by the solution of the bosonic model, as we have claimed several times before.

\section{Casimir decomposition of the state space}

The central result of the following section can be considered as a corollary of our observation that the ground states of the field theory all transform according to projective representations. As we shall explain below, this implies that one can count the number of field theoretic states in any given $\operatorname{psl}(2 \mid 2)$ representation through some variant of the Racah-Speiser algorithm. The results play a central role for the study of the RR deformation. Though we shall publish this investigation in a separate paper, we decided to include 
a short discussion of the RR deformation and its relation to Casimir decompositions at the end of this section.

\subsection{The $\operatorname{psl}(2 \mid 2)$ symmetry and its branching functions}

By construction, the RR deformation preserves both global left and right psl(2|2) action. Hence, the state space of the perturbed theory will continue to carry a representation of these two commuting $\operatorname{psl}(2 \mid 2)$ transformations. In order to study the perturbation, it seems worthwhile to decompose the state space of the model explicitly with respect to the preserved symmetries, in particular with respect to the left and right $\operatorname{psl}(2 \mid 2)$ action. Since this is a bit cumbersome to write down for the full state space of the bulk theory, we shall explain the main idea in a simpler example that is relevant for the study of instantonic point-like branes in the $\operatorname{PSU}(1,1 \mid 2)$ model.

Naively, one might expect that the spectrum of open strings on such branes contains no zero modes and hence possesses a unique ground state that transforms in the trivial representation of the preserved $\operatorname{psl}(2 \mid 2)$ of the boundary theory. If this was true, the decomposition of the boundary spectrum into representations of $\operatorname{psl}(2 \mid 2)$ would be extremely difficult, if not impossible. In fact, states of the boundary theory would then transform according to all those representations that appear in some tensor power of the adjoint representation. Our investigations show [32] that very exotic indecomposable representations can emerge in this way. Since the adjoint representation of $\operatorname{psl}(2 \mid 2)$ is atypical and not projective, tensor powers are specifically not decomposable into projectives. The indecomposables that arise in this way cannot even be listed easily so that the bookkeeping of the possible states and their transformation laws appears as a daunting task.

Fortunately, the boundary spectrum of a maximally symmetric point-like brane in our WZNW model does possess zero modes. In fact, a thorough investigation of the gluing condition shows that while such branes are localized in the bosonic coordinates they must necessarily be delocalized in all fermionic directions (details will be published elsewhere). Since there are eight fermionic coordinates, each contributing one zero mode, we conclude that the ground states transform according to the representation

$$
B(0,0) \cong \mathcal{P}_{0} \oplus[1,0] \oplus[0,1]
$$

i.e. as a sum of projective representations. Excited states therefore transform in represen-

tations that emerge from a product of a projective representation with some power of the 
adjoint and which, by abstract mathematical results, can be decomposed into projectives. This result is once more a confirmation of what we saw in the bulk theory: the physical states of the PSU $(1,1 \mid 2)$ WZNW model all transform in projective representations, i.e. they either form typical long multiplets or they sit in maximally extended atypical representations.

In the first part of this work we listed explicitly all the (finite dimensional) projective representations of $\operatorname{psl}(2 \mid 2)$. Our aim now is to compute the branching functions for a state space of the WZNW model into $\operatorname{psl}(2 \mid 2)$ representations. We shall first show that there is an efficient algorithm that determines this branching explicitly and then we shall state the results for one example. Note that the branching functions can be considered as characters of the so-called Casimir algebra [19].

\subsubsection{The Racah-Speiser algorithm}

In its original form, the Racah-Speiser algorithm is a powerful tool which allows to decompose tensor products of representations of semi-simple Lie algebras. The only knowledge required is the weight content of one of the representations involved and the action of the Weyl group. In this paper we will use it in a slightly different setup. We assume that we have given a set of weights belonging to some finite dimensional representation $R$. The weight content can be encoded in some generating function, the character of $R$. In the case of ordinary bosonic Lie algebras $R$ can be written as a direct sum of irreducible representations $R_{i}$. The Racah-Speiser algorithm allows us to determine the $R_{i}$ and their multiplicities by just analyzing the original weight system. Consequently, the character of $R$ can be expressed through the characters of the irreducible representations $R_{i}$.

It is clear that for principal reasons the algorithm cannot be extended to Lie superalgebras. This is due to the presence of not fully reducible representations: there can exist several inequivalent representations which have the same weight content. Our claim, however, is that the Racah-Speiser algorithm may be extended to Lie superalgebras as long as one is just dealing with projective representations. In fact, projective representations share the crucial property that their characters always contain the fermionic factor $V_{F}$ (see eq. (2.7) ) multiplied by some representations of the bosonic subalgebra. Hence, the

problem of reconstructing the representation content of a projective representation out of its weight system is reduced to a problem concerning the bosonic subalgebra. This statement also allows to calculate tensor products of projective representations using character 
methods. One could thus say that indecomposable projective representations - typical irreducibles and projective covers - play a similar role for (simple) Lie superalgebras as irreducible ones play for ordinary (simple) bosonic Lie algebras.

Before we start to discuss complications arising in the super case it is convenient to explain the idea of the original Racah-Speiser algorithm in the example of the Lie algebra $\operatorname{sl}(2)$. Suppose we are given some (finite dimensional) representation $R$ of $\operatorname{sl}(2)$ with a character of the form

$$
\chi_{R}(z)=\sum_{2 l \in \mathbb{Z}} a_{l} z^{l} .
$$

The coefficients $a_{l}$ give the multiplicity of states with isospin $l$. For consistency they have to satisfy $a_{l}=a_{-l}$. It is easy to see that we may rewrite the previous expression in terms of characters of irreducible representations as

$$
\chi_{R}(z)=\sum_{j \geq 0}\left[a_{j}-a_{j+1}\right] \chi_{j}(z)
$$

Due to the linearity of the problem it is enough to prove this relation on the level of characters of irreducible representations where it is obvious. Basically the formula counts the number of weights with isospin $j$ and checks how many still exist for $j+1$. The latter obviously do not belong to the spin $j$ representation and have to be subtracted. The Racah-Speiser trick provides a very simple way e.g. to derive the Casimir characters of $\widehat{\mathrm{su}}(2)_{k}$, see [43], after splitting the algebra into parafermions and a $\hat{u}(1)$ part.

In this paper we are interested in the Casimir characters of $\widehat{\operatorname{psl}}(2 \mid 2)_{k}$. As we will see in the following section this problem may be reduced to one solely involving the bosonic subalgebra $\operatorname{sl}(2) \oplus \operatorname{sl}(2)$. Applying formula (7.2) to this new situation with two factors we find

$$
\begin{aligned}
\chi_{R}\left(z_{1}, z_{2}\right) & =\sum_{2 l_{1}, 2 l_{2} \in \mathbb{Z}} a_{l_{1} l_{2}} z_{1}^{l_{1}} z_{2}^{l_{2}} \\
& =\sum_{j_{1}, j_{2} \geq 0}\left[a_{j_{1}, j_{2}}-a_{j_{1}+1, j_{2}}-a_{j_{1}, j_{2}+1}+a_{j_{1}+1, j_{2}+1}\right] \chi_{j_{1}}\left(z_{1}\right) \chi_{j_{2}}\left(z_{2}\right) .
\end{aligned}
$$

In the intended application of this formula to affine modules the multiplicities of the weights are infinite. In that case we let the coefficients $a_{l_{1} l_{2}}$ depend on a formal variable $q$ in order to be able to distinguish the energy of the states and to resolve the infinities.

The previous formulas are obvious even without using fancy technology and recruiting great names. Yet, since our ideas for the calculation of Casimir characters are likely to 
apply for more complicated Lie superalgebras we would like to make clear from the start that our considerations easily may be generalized. In that case one has to use the shifted action of the Weyl group $w \cdot \mu=w(\mu+\rho)-\rho$ in order to map the given weights into the fundamental domain, taking into account the sign of the transformation. The result will then be a sum over the corresponding highest weight vectors, and these in turn can be replaced by characters of irreducible representations.

\subsubsection{Example: Branching rules for $\mathcal{P}_{0}$}

As we have announced before, our goal is to decompose the space of physical states of the $\operatorname{PSU}(1,1 \mid 2)$ WZNW model with respect to the horizontal subalgebra. For simplicity we shall focus on one particular building block of the open string spectrum on a point-like brane, namely on the decomposition of the representation $\hat{\mathcal{P}}_{0}$ of the affine $\widehat{\operatorname{psl}}(2 \mid 2)$. The results can easily be extended to the other two pieces $[0,1]^{\wedge}$ and $[1,0]^{\wedge}$ which appear in the brane's spectrum (see above) 14

The representation $\hat{\mathcal{P}}_{0}$ is built on top of the finite dimensional projective cover $\mathcal{P}_{0}$ by acting with the negative modes of the current algebra, followed by the removal of all bosonic singular vectors, i.e. all the singular vectors that do not appear among the ground states. As we observed above the states at higher energy levels transform in the tensor product of $\mathcal{P}_{0}$ with (symmetrized) tensor products of the adjoint representation of $\operatorname{psl}(2 \mid 2)$ with itself. Since the tensor products of arbitrary representations with a projective one are projective again, the affine representation may be written as

$$
\left.\hat{\mathcal{P}}_{0}\right|_{\mathrm{psl}(2 \mid 2)}=\sum_{j_{1} \neq j_{2}} a_{j_{1} j_{2}}(q)\left[j_{1}, j_{2}\right]+\sum_{j} b_{j}(q) \mathcal{P}_{j}
$$

This should be read as a formal decomposition of the affine representation into representations of the horizontal subalgebra. The multiplicities on each energy level are contained in the generating functions $a_{j_{1} j_{2}}(q)$ and $b_{j}(q)$ which can be considered as characters of the Casimir algebra.

Now we employ our knowledge about the bosonic content of projective representations as stated in eq. (2.23). If we denote by $\chi_{F}$ the character of the fermionic zero modes, as before, then the character of $\hat{\mathcal{P}}_{0}$ is given by

$$
\chi_{\hat{\mathcal{P}}_{0}}=\left[\sum_{j_{1} \neq j_{2}} a_{j_{1} j_{2}}(q) \chi_{\left(j_{1}, j_{2}\right)}+\sum_{j} b_{j}(q)\left[2 \chi_{(j, j)}-\chi_{\left(j+\frac{1}{2}, j+\frac{1}{2}\right)}-\chi_{\left(\left|j-\frac{1}{2}\right|,\left|j-\frac{1}{2}\right|\right)}\right]\right] \chi_{F} .
$$

\footnotetext{
${ }^{14}$ It is also rather straightforward to find the generalization to infinite dimensional representations like those that appear in the bulk spectrum.
} 
All the relevant notations have been introduced in the first part of this work. Given the supercharacter on the left hand side we can then in principle derive the coefficients $a_{j_{1} j_{2}}(q)$ and $b_{j}(q)$ using a refined version of the Racah-Speiser algorithm. From part I of this work we recall that

$$
\chi_{\hat{\mathcal{P}}_{0}}\left(q, z_{1}, z_{2}\right)=2 \chi_{[0,0]}\left(q, z_{1}, z_{2}\right)-2 \chi_{[1 / 2,1 / 2]}\left(q, z_{1}, z_{2}\right)
$$

Explicit formulas for the supercharacters on the right hand side were provided in eq. (3.25). What is most important for us is that the supercharacter of $\hat{\mathcal{P}}_{0}$ possesses an overall factor $\chi_{F}$ as any projective representation. By expansion into powers of $z_{1}$ and $z_{2}$ and comparison we are thus able to uniquely determine the functions $a_{j_{1} j_{2}}(q)$ and $b_{j}(q)$. To this end let us consider the previous expression as a generating function for the $q$-series $c_{m n}(q)$,

$$
\chi_{\hat{\mathcal{P}}_{0}}\left(q, z_{1}, z_{2}\right)=\sum_{2 m, 2 n \in \mathbb{Z}} c_{m n}(q) z_{1}^{m} z_{2}^{n} \chi_{F}\left(z_{1}, z_{2}\right) \text {. }
$$

Then the functions $a_{j_{1} j_{2}}(q)$ are determined by the Racah-Speiser trick,

$$
a_{j_{1} j_{2}}(q)=c_{j_{1}, j_{2}}(q)-c_{j_{1}+1, j_{2}}(q)-c_{j_{1}, j_{2}+1}(q)+c_{j_{1}+1, j_{2}+1}(q)
$$

These quantities also have meaning for $j_{1}=j_{2}$. Yet, in that case they do not count "real" representations but just the Kac modules which sit inside the projective representations. We find

$$
a_{00}=2 b_{0}+b_{\frac{1}{2}} \quad a_{\frac{1}{2} \frac{1}{2}}=2 b_{0}+2 b_{\frac{1}{2}}+b_{1} \quad a_{j j}=b_{j-\frac{1}{2}}+2 b_{j}+b_{j+\frac{1}{2}} .
$$

This relation needs to be inverted to find the values of the $b_{j}$. For a fixed energy level this inversion is almost trivial, one just has to start from the contributions with highest spin in order to find the corresponding projective covers. The described procedure may seem a bit abstract, but it is straightforward to implement the expansions and the Racah-Speiser trick on the computer. In this way the branching functions $a(q)$ and $b(q)$ can in principle be determined to any desired order. For large values of the level $k$ one finds for instance

$$
\begin{aligned}
& \hat{\mathcal{P}}_{0}(q)= \mathcal{P}_{0} \oplus q\left[4 \mathcal{P}_{\frac{1}{2}} \oplus 6([1,0] \oplus[0,1]) \oplus 2\left(\left(\frac{3}{2}, \frac{1}{2}\right) \oplus\left(\frac{1}{2}, \frac{3}{2}\right)\right)\right] \\
& \oplus q^{2}\left[\mathcal{P}_{0} \oplus 16 \mathcal{P}_{\frac{1}{2}} \oplus 4 \mathcal{P}_{1} \oplus 4([2,1] \oplus[1,2]) \oplus 6([2,0] \oplus[0,2])\right. \\
&\left.\oplus 24([1,0] \oplus[0,1]) \oplus 2\left(\left(\frac{5}{2}, \frac{1}{2}\right) \oplus\left(\frac{1}{2}, \frac{5}{2}\right)\right) \oplus 18\left(\left(\frac{3}{2}, \frac{1}{2}\right) \oplus\left(\frac{1}{2}, \frac{3}{2}\right)\right)\right] \oplus \cdots
\end{aligned}
$$

With a bit more work one might also be able to write down closed formulas. 
Before we conclude this subsection, let us stress once more that the whole procedure relied extremely on the fact that only projective representations occurred in the affine character. If there had been non-projective atypicals in addition to the projective ones, knowledge of the characters would have been insufficient to determine the decomposition.

\subsection{Some remarks on the $\mathrm{RR}$ deformation}

The RR deformation of the $\mathrm{AdS}_{3}$ background corresponds to adding the the following extra to the action of the WZNW model

$$
\mathcal{S}_{R R ; \lambda}^{\mathrm{PSL}(2 \mid 2)}=-\frac{\lambda}{2 \pi} \int d^{2} z \operatorname{tr}\left(S^{-1} \partial S S^{-1} \bar{\partial} S\right) .
$$

We can rewrite the perturbation in terms of the fields we have studied above. To this end, we shall need the left and right invariant (anti-)holomorphic currents $J^{\mu}(z)$ and $\bar{J}^{\nu}(\bar{z})$ along with some degenerate primary fields $\Phi_{\mu \nu}(z, \bar{z})$ that transform in the (atypical) adjoint representation $[1 / 2]$ of $\operatorname{psl}(2 \mid 2)$, i.e.

$$
\begin{aligned}
J^{\mu}(z) \phi_{\nu \rho}(w, \bar{w}) & =\frac{i f^{\mu \nu \sigma}}{z-w} \phi_{\sigma \rho}(w, \bar{w})+\ldots, \\
\bar{J}^{\mu}(\bar{z}) \phi_{\nu \rho}(w, \bar{w}) & =\frac{i f^{\mu \rho \sigma}}{\bar{z}-\bar{w}} \phi_{\nu \sigma}(w, \bar{w})+\ldots .
\end{aligned}
$$

It is then easy to identify the RR perturbation with the one that is generated by the composite field

$$
\Phi(z, \bar{z})=: J^{\mu}(z) \phi_{\mu \nu}(z, \bar{z}) \bar{J}^{\nu}(\bar{z}):
$$

By construction, the field $\Phi$ has conformal weights $h=\bar{h}=1$ in the WZNW model but in principle its dimension could change when we perturb the theory. According to [18] (see also [44, 45] for related studies), however, $\Phi$ is truly marginal, i.e. its dimension remains at $h=\bar{h}=1$ in all orders of perturbation theory. Note that the perturbation with the field $\Phi$ rescales the kinetic term of the WZNW model and thus alters the relative normalization of kinetic and Wess-Zumino term. The resulting moduli space and its physical interpretation is summarized in figure 1 .

We do not want to re-derive the conformal invariance of the RR deformation here, but instead will discuss a somewhat more general assertion. To begin with, it follows from the marginality of $\Phi$ that the deformed theory possesses the usual (anti-)holomorphic Virasoro field. The latter is $\operatorname{psl}(2 \mid 2)$ invariant and hence acts as a symmetry within the branching spaces of the decomposition (7.4). The true chiral symmetry of the deformed 


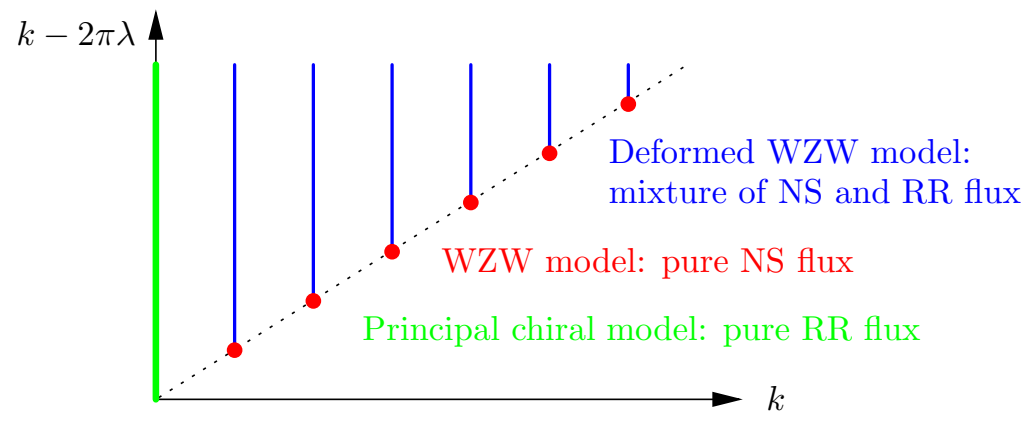

Figure 1: The moduli space of string theory on PSU $(1,1 \mid 2)$. The vertical axis gives the normalization of the kinetic term, the horizontal the normalization of the Wess-Zumino term. The lines with $\lambda \neq 0$ correspond to marginal deformations of the WZNW model.

theory is larger. In fact, it was claimed in [18] that all the Casimir fields in the current algebra provide chiral fields of the deformed model, i.e. that all fields of the form

$$
W^{(n)}=t_{\mu_{1} \ldots \mu_{n}} J^{\mu_{1}} \ldots J^{\mu_{n}}
$$

involving contractions with an invariant, symmetric and traceless tensor $t$ are holomorphic even after a finite deformation with $\Phi$. We would like to establish this in first order perturbation theory. Let us emphasize that the symmetry and tracelessness of $t$ imply that on the right hand side no particular normal ordering prescription has to be specified.

To begin with, we analyze the behaviour of a single current. An insertion of the latter into a correlation function corresponds to the perturbative expansion

$$
\left\langle J^{\mu}(z, \bar{z}) \cdots\right\rangle_{\lambda}=\left\langle J^{\mu}(z) \cdots\right\rangle_{0}-\lambda\left\langle J^{\mu}(z) \cdots \int d^{2} w \Phi(w, \bar{w})\right\rangle_{0}+\cdots .
$$

The integral may easily be evaluated with the help of the operator product expansion

$$
J^{\mu}(z) \Phi(w, \bar{w})=\frac{k: \phi_{\nu}^{\mu} \bar{J}^{\nu}:(w, \bar{w})}{(z-w)^{2}}=\frac{k: \phi^{\mu}{ }_{\nu} \bar{J}^{\nu}:(z, \bar{z})}{(z-w)^{2}}-\frac{k: \partial \phi_{\nu}^{\mu} \bar{J}^{\nu}:(z, \bar{z})}{z-w} .
$$

The second expansion where the argument $w$ is replaced by $z$ is more suitable for the calculation of the integral. Introducing the usual step function cut-off which restricts the integration to the domain $|z-w|^{2}>a^{2}$ we find, following an argument of Cardy [46],

$$
\bar{\partial} J^{\mu}=\pi \lambda k: \partial \phi^{\mu}{ }_{\nu} \bar{J}^{\nu}:+\cdots=-2 \pi i \lambda k f_{\rho \sigma}^{\mu}: J^{\rho} \phi^{\sigma}{ }_{\nu} \bar{J}^{\nu}:+\cdots .
$$

This equation is the quantum analogue of the classical relation $\bar{\partial} J=c(k, \lambda)\left[J, g \bar{J} g^{-1}\right]$ which can be derived from the equations of motion of the perturbed Lagrangian in connection with the Maurer-Cartan equation. We believe that the higher order terms in $\lambda$ 
reproduce precisely the classical equation, i.e. that $\bar{\partial} J^{\mu}$ is proportional to $f_{\rho \sigma}^{\mu}: J^{\rho} \phi^{\sigma}{ }_{\nu} \bar{J}^{\nu}$ : and that the $\lambda$-dependent prefactor coincides with the classical expression $c(k, \lambda)$.

We are now finally prepared to investigate the chiral properties of the Casimir currents $W^{(n)}$. Taking the derivative will produce a number of terms but we can move the current with the derivative to the last position. This is because the difference between two different normal orderings of the currents and their derivatives always involves the metric or the structure constants with two open indices. Upon contraction with $t$ these terms vanish by assumption. Together with the result (17.16) this implies

$$
\bar{\partial} W^{(n)}=-2 \pi i \lambda k f_{\rho \sigma}^{\mu_{n}} t_{\mu_{1} \cdots \mu_{n}} J^{\mu_{1}} \cdots J^{\mu_{n-1}} J^{\rho} \phi_{\nu}^{\sigma} \bar{J}^{\nu}
$$

In this equation the normal ordering again is not relevant. This is basically due to the same reasons as above, combined with the fact that operator products of currents with the field $\phi$ give rise to a single term involving the structure constants $f$. In addition we have to use the vanishing of the dual Coxeter number of $\operatorname{psl}(2 \mid 2)$ in order to get rid of multiple $f$-contractions. Reordering and relabeling the currents we then find the desired result $\bar{\partial} W^{(n)}=0$ due to the invariance of the tensor $t$.

Having established the holomorphicity of Casimir fields, it seems important to add a few comments on denominations. It would be tempting to baptize the chiral algebra that is generated by the Casimir fields as Casimir algebra. But this is not how the latter term is used. By definition, the $\operatorname{psl}(2 \mid 2)$ Casimir algebra is the chiral algebra whose characters are given by the branching functions we computed in the previous subsection [47, 48]. These are not the same as the characters of the chiral algebra that is generated by the Casimir fields [19]. In fact, characters of the latter may be calculated using the quantum Drinfeld-Sokolov reduction [49, 50, and they only appear as building blocks for the branching functions above, but in most cases the precise relation is highly involved. Put differently, the chiral algebra generated by the Casimir fields is much smaller than the Casimir algebra. Roughly, the difference is that the Casimir fields are obtained as invariant combinations of currents whereas the Casimir algebra also contains invariant fields involving derivatives of currents. The first field in the Casimir algebra that is not a Casimir field appears at conformal weight $h=4$ and it is given by $\operatorname{str}(\partial J \partial J)$. In this sense, the decompositions discussed in the previous subsection would not seem sufficient in order to control the deformation away from the WZNW point. On the other hand, the spectra possess a higher degree of degeneracy which cannot be explained by the chiral symmetries we have described. Take, for example, the current itself: it does 
not stay holomorphic beyond the WZ point, yet its conformal weight rests at $h=1$ all along the line of marginal deformations. Such additional degeneracies may possibly be attributed to some new symmetry whose algebraic structure remains to be uncovered 15 In a forthcoming paper, we shall suggest a different picture that is intimately related to Casimir algebras.

\section{Outlook and conclusions}

In this work we presented a complete solution of the WZNW model on the supergroup $\operatorname{PSU}(1,1 \mid 2)$ in terms of a free fermion construction which kept the bosonic symmetry manifest. After a thorough discussion of the representation theoretic foundations in part I we derived the precise form of the spectrum based on methods of harmonic analysis. It was found that the state space splits into two parts. The typical (non-BPS) sector behaves nicely and decomposes into tensor products of chiral irreducible representations. On the other hand, there exists an atypical (BPS) sector related to states with vanishing conformal dimension where our intuition from purely bosonic WZNW models breaks down. Here we found non-chiral indecomposable representations on which the zero-mode of the energy momentum tensor is not diagonalizable. Although we just provided a brief sketch of how correlation functions may be calculated it is thus established that the $\operatorname{PSU}(1,1 \mid 2)$ WZNW model is a logarithmic conformal field theory.

We would like to stress that the logarithms only arise because the WZNW model on the supergroup $\mathrm{PSU}(1,1 \mid 2)$ does not factorize into a product of fermionic and bosonic subsectors, contrary to a widespread claim. In the minisuperspace theory, the coupling may be ascribed to the existence of the nilpotent term $Q$ in the Laplacian. The operator $Q$ is a differential operator in the fermionic directions with coefficients which vary along the bosonic base. Compared to the factorized theory, this additional term alters the structure of the eigenfunctions along with their transformation law under $\operatorname{psl}(2 \mid 2)$. As a result, all the states transform in projective representations only, both in the particle limit and the full field theory. It is worthwhile to emphasize that without the coupling the field theory ground states would have transformed as (a product of) Kac modules, even in the atypical sector where the modules degenerate. Hence, the interaction between bosons and fermions drastically changes the embedding structure of the fermionic singular vectors in

\footnotetext{
${ }^{15}$ Additional Yangian symmetries (see [51, 52 for a review) are known to exist in these models, see e.g. [53. See also [54, 55, 56, 57, 58, 59, 60] for some closely related studies and results.
} 
our state space.

As in usual free field constructions for purely bosonic WZNW models one might be tempted to set all singular vectors to zero and to work with irreducible representations only. But in the case of supergroup WZNW models, the fermionic singular vectors cannot be decoupled, at least for generic values of the level $k$. An investigation of the relevant Knizhnik-Zamolodchikov equations similar to the one in [21] shows indeed that generically local solutions contain logarithms. Hence, at least some of the fermionic singular vectors are needed for consistency. There are also other ways to argue that fermionic singular vectors have a very different status from their bosonic counterparts. In particular, it is unavoidable that the states on higher energy levels in affine modules - irreducible or not - transform in reducible but indecomposable representations of the horizontal superalgebra. Therefore it seems unnatural to insist on removing fermionic singular vectors among the ground states and to work with irreducible affine representations only. Even worse, in atypical irreducible affine modules or the associated Kac modules we have little or no chance to ever control the behavior of all excited states under global psu( $1,1 \mid 2)$ transformations.

It is in this context that projectivity of representations comes to our rescue. Indeed, for affine modules based on a projective representation of $\operatorname{psu}(1,1 \mid 2) \oplus \operatorname{psu}(1,1 \mid 2)$ all the excited states transform in projective representations. Moreover, the Racah-Speiser algorithm allows to determine the decomposition rather explicitly. Since the psu(1,1|2) symmetry is an important part of the symmetry that remains unbroken when we turn on RR flux, the occurrence of projective representations is the best we could hope for if we are interested in getting a handle on the $\sigma$-model describing $\operatorname{AdS}_{3} \times \mathrm{S}^{3}$ with a mixture of NS and RR fluxes. Hence, we think that even in cases where it might not be strictly necessary for reasons of consistency it is much preferable to define the WZNW model such that it includes all the fermionic singular vectors. This is also suggested by the minisuperspace analysis in which fermionic singular vectors appear naturally among the eigenstates of the Laplacian.

Another remarkable consequence of the coupling of bosons and fermions is that the naive algebra of functions on $\operatorname{PSU}(1,1 \mid 2)$ - generated by the product of functions on its body $\mathrm{AdS}_{3} \times S^{3}$ with monomials in the fermionic variables - does not furnish the appropriate model for the representation space of the global supersymmetry transformations. The origin of this surprising fact is that the fermions transform in a finite dimensional 
non-unitary representation of the bosonic subgroup. In fact, along with the two supersymmetry transformation laws - one for the decoupled theory and one for the full theory - we have to distinguish two different models for the representation spaces. While in the decoupled theory we are indeed working with the naive algebra of functions, this is not true anymore in the full, coupled system. The operator $\Xi$ derived from $Q$ (or analogously the interaction term 16 in the full WZW model) mediates between these two inequivalent representation spaces. As part of this process the operator $\Xi$ entangles the two chiral subsectors of the free fermion theory by adding subleading contributions to the free field vertex operators. These in turn change the normalizability properties with respect to the bosonic subgroup and explain why the naive algebra of functions did not provide the proper representation space for the full theory.

Though the observations we have listed in the last few paragraphs emerged from the study of the WZNW model on $\operatorname{PSU}(1,1 \mid 2)$, it is clear that they extend to a much larger class of models. Let us point out that the minisuperspace analysis is not at all restricted to the WZ-point and hence most of our remarks concerning the structure and importance of fermionic singular vectors apply to more general sigma models, in particular to principal chiral models on a large class of supergroups. Similarly, subtleties such as the coupling of bosonic and fermionic degrees of freedom - which eventually lead to the occurrence of indecomposable non-chiral representations and logarithmic correlation functions - are bound to arise in more general setups. In fact, the same features are common to most relativistic theories with a global target space superalgebra symmetry.

Supergroups and cosets thereof appear naturally in all attempts to quantize superstring theory in a manifestly target space supersymmetric way. On the corresponding backgrounds, the supersymmetry transformations are realized geometrically as an isometry or, more precisely, as the left and (for the group case) right action of the supergroup on itself. This statement holds in particular for all supersymmetric AdS-spaces which can be expressed as (right) cosets based on superconformal groups such as $\operatorname{PSU}(N, N \mid 2 N)$ [2, [5], higher dimensional relatives of the supergroup considered here. Due to the presence of Ramond-Ramond fluxes the quantization of these backgrounds has been a notoriously difficult task.

In the case of $A d S_{3}$ backgrounds, the hybrid approach of [4] provides one way to resolve the conceptual issues. As we stressed several times before, it involves the sigma

\footnotetext{
${ }^{16}$ One could also refer to it as screening charge but it should be kept in mind that no BRST procedure is implied in our description.
} 
model on $\operatorname{PSU}(1,1 \mid 2)$. Concerning higher dimensional backgrounds, the quantization of string backgrounds using pure spinors [6] may be considered the most promising recent development, see also [61, 62 for some results on $\mathrm{AdS}_{5}$ backgrounds that were obtained in this formalism. Yet, one drawback of the original formulation was the necessity of solving the pure spinor constraint explicitly using a suitable choice of coordinates. This in turn partly ruined the manifest Lorentz covariance. The problem was overcome in [63] upon introduction of new additional ghost systems. In follow up papers the central role of a special affine Lie superalgebra has been emphasized [64, 65] (see also [66]). The existence of local and global superalgebra symmetries connects these developments with the technical aspects of our work, even though we have not been concerned with imposing the physical state conditions.

\section{Acknowledgements}

We would like to thank Giuseppe d'Appollonio, Denis Bernard, Matthias Gaberdiel, Jérôme Germoni, Niall MacKay, Yaron Oz, Soo-Jong Rey, Sylvain Ribault, Hubert Saleur, Kareljan Schoutens, Didina Serban, Vera Serganova, Paul Sorba, Anne Taormina, Jörg Teschner, Alexei Tsvelik, Gérard Watts, Kay Wiese and Charles Young for useful and inspiring discussions during various stages of this project. We are also grateful for the hospitality at the ESI during the workshop "String theory in curved backgrounds" which stimulated the present work. Moreover, Thomas Quella acknowledges the kind hospitality of the SPhT in Saclay and at the DESY in Hamburg during numerous visits.

This work was partially supported by the EU Research Training Network grants "Euclid", contract number HPRN-CT-2002-00325, "Superstring Theory", contract number MRTN-CT-2004-512194, and "ForcesUniverse", contract number MRTN-CT-2004005104. Until September 2006 Thomas Quella has been funded by a PPARC postdoctoral fellowship under reference PPA/P/S/2002/00370. He also received partial support by the PPARC rolling grant PP/C507145/1. 


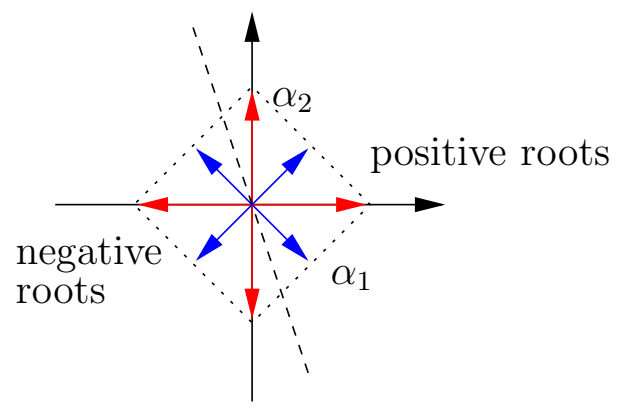

Figure 2: The root diagram of $\operatorname{psl}(2 \mid 2)$.

\section{A On the irreducibility of generalized Fock spaces}

The Kac-Kazhdan formula encodes the precise structure of singular vectors in a Verma module, including their multiplicities [67, 68]. This in turns allows one to represent the characters of irreducible representations as alternating sums of characters of Verma modules [69]. In this section we are going to discuss the singular vectors of Verma modules over $\widehat{\operatorname{psl}}(2 \mid 2)$ and show that their irreducible quotients are typically isomorphic to the generalized Fock modules introduced in section 3.2. Instead of working directly with the Kac-Kazhdan determinant, we are taking a more direct and physically more intuitive route here, which to the best of our knowledge should be completely equivalent. Nevertheless we have to introduce a bit of notation first.

The set of all pairs $\left(\mu_{1}, \mu_{2}\right)$ with $\mu_{i} \in \mathbb{Z}$ forms the weight lattice of $\operatorname{psl}(2 \mid 2)$. The two entries correspond to weights in the individual factors of $\operatorname{sl}(2) \oplus \operatorname{sl}(2)$, respectively (let us recall that the weight is twice the spin). Due to the embedding into the supersymmetric setup we have the slightly unusual scalar product

$$
\left\langle\left(\lambda_{1}, \lambda_{2}\right),\left(\mu_{1}, \mu_{2}\right)\right\rangle=-\frac{1}{2}\left(\lambda_{1} \mu_{1}-\lambda_{2} \mu_{2}\right) .
$$

In order to describe the root system of the Lie superalgebra $\operatorname{psl}(2 \mid 2)$ at least one of the two simple roots has to be chosen fermionic. It turns out to be useful to work with a root system whose simple roots correspond to $\alpha_{1}=(1,-1)$ and $\alpha_{2}=(0,2)$. The remaining positive roots are then given by $\alpha_{1}+\alpha_{2}=(1,1)$ and $2 \alpha_{1}+\alpha_{2}=(2,0)$. The fermionic roots have multiplicity two while the bosonic ones just have multiplicity one. A sketch of the root diagram can be found in figure 2 .

If we denote the Weyl vector as $\rho=(1,1)$ as usual then the conformal dimension of a 
highest weight representation $\mu$ is given by

$$
h_{\mu}=\frac{\langle\mu, \mu+2 \rho\rangle}{2 k} .
$$

Note that the conformal dimension is invariant under the transformation

$$
w * \mu=w(\mu+\rho)-\rho
$$

where $w$ refers to an element of the bosonic Weyl group, i.e. a pair of elements of the Weyl group of $\mathrm{sl}(2)$ (the statement holds in general though). This may be traced back to the fact that the corresponding Weyl transformations leave the metric $\langle\cdot, \cdot\rangle$ invariant. Reflections perpendicular to the fermionic roots, however, change the sign. Therefore they should not be used in the formula above.

After these remarks we are finally prepared to discuss the structure of Verma modules over $\widehat{\operatorname{psl}}(2 \mid 2)$. We will consider a Verma module that is based on a highest weight $\left(\mu, h_{\mu}\right)$ where we included the eigenvalue of $L_{0}$, the conformal weight, for completeness. Singular vectors $\left(\nu, h_{\nu}\right)$ can just occur if the difference $\left(\mu-\nu, h_{\mu}-h_{\nu}\right)$ is a linear combination of affine simple roots with non-negative coefficients. This in particular implies that the conformal weights have to satisfy the relation

$$
h_{\nu}=h_{\mu}+n
$$

with a non-negative integer $n$. Note that the conformal dimension of a singular vector indeed is fixed to be $h_{\nu}$ as in (A.2) for algebraic reasons and cannot be chosen arbitrarily. Moreover, the difference $\mu-\nu$ has to be an element of the root lattice of $\operatorname{psl}(2 \mid 2)$ (in fact the actual condition is more restrictive). In the following we will assume that every weight which according to the previous criteria has the potential to describe a singular vector in fact is singular. This seems to be a straightforward consequence of the Kac-Kazhdan formula [67, 68]. It is even enough to restrict the analysis to the case where the affine weights differ by a multiple of a simple root. The other states which decouple are just descendents of the ones obtained through the latter.

To illustrate the decoupling conditions we have to specify the affine simple roots first. The bosonic simple roots are given by the set

$$
\left\{(\alpha,-n) \mid \alpha \in \Delta^{(0)}, n>0\right\} \cup\left\{\left(\alpha_{2}, 0\right)\right\} .
$$

The second label in each tupel refers to the energy of the roots, i.e. to the mode number. The remaining simple roots are fermionic,

$$
\left\{(\alpha,-n) \mid \alpha \in \Delta^{(1)}, n>0\right\} \cup\left\{\left(\alpha_{1}, 0\right)\right\} .
$$


In the previous definitions $\Delta^{(0)}$ and $\Delta^{(1)}$ refer to the bosonic and fermionic roots of $\operatorname{psl}(2 \mid 2) . \quad \alpha_{1}$ and $\alpha_{2}$ have been specified above. Let us stress that they do not coincide with the simple roots of $\operatorname{sl}(2) \oplus \operatorname{sl}(2)$.

Let us discuss the bosonic decoupling conditions first. For the $m$-fold application of the root $(( \pm 2,0), n)$ the decoupling condition reads

$$
h_{\left(\mu_{1} \pm 2 m, \mu_{2}\right)} \stackrel{!}{=} h_{\left(\mu_{1}, \mu_{2}\right)}+m n \quad \Rightarrow \quad \mp\left(\mu_{1}+1\right)=n k+m .
$$

This equation cannot be solved for $m$ (for positive level $k$ and due to the restrictions on $\mu_{1}$ ), thus proving the absence of bosonic singular vectors with respect to the first factor $\widehat{\mathrm{sl}}(2)_{-k}$. On the other hand the $m$-fold application of the root $((0, \pm 2), n)$ yields

$$
h_{\left(\mu_{1}, \mu_{2} \pm 2 m\right)} \stackrel{!}{=} h_{\left(\mu_{1}, \mu_{2}\right)}+m n \quad \Rightarrow \quad \pm\left(\mu_{2}+1\right)=n k-m .
$$

In this case the equation may always be solved for $m$ (for positive level $k$ ). Consequently, all Verma modules of $\widehat{\operatorname{psl}}(2 \mid 2)_{k}$ possess bosonic singular vectors.

The situation is different for the fermionic simple roots because they just may be applied once, i.e. $m$ is bound to be one. The corresponding four decoupling conditions are

$$
\begin{aligned}
& h_{\left(\mu_{1}+1, \mu_{2}+1\right)} \stackrel{!}{=} h_{\left(\mu_{1}, \mu_{2}\right)}+n \quad \Rightarrow \quad \mu_{1}-\mu_{2}=-2 n k \\
& h_{\left(\mu_{1}+1, \mu_{2}-1\right)} \stackrel{!}{=} h_{\left(\mu_{1}, \mu_{2}\right)}+n \quad \Rightarrow \quad \mu_{1}+\mu_{2}+2=-2 n k \\
& h_{\left(\mu_{1}-1, \mu_{2}+1\right)} \stackrel{!}{=} h_{\left(\mu_{1}, \mu_{2}\right)}+n \quad \Rightarrow \quad \mu_{1}+\mu_{2}+2=2 n k \\
& h_{\left(\mu_{1}-1, \mu_{2}-1\right)} \stackrel{!}{=} h_{\left(\mu_{1}, \mu_{2}\right)}+n \quad \Rightarrow \quad \mu_{1}-\mu_{2}=2 n k \text {. }
\end{aligned}
$$

In this case none of these equations necessarily possesses a solution. We thus realize that the existence of fermionic singular vectors is a rather special incidence, related to the factual absence of the variable $m$. It is thus sensible to introduce the notion of a typical Verma module. This is a Verma module in which none of the fermionic vectors decouples. In other words: The highest weight has to violate all the conditions (A.9a - (A.9d).

The analysis above has to be slightly refined for $n=0$. The reason is that for $n=0$ we are bound to use the positive roots of $\operatorname{psl}(2 \mid 2)$ in the equations above but the negative ones have to be discarded. Thus just half of the equations above will correspond to a valid decoupling condition under these circumstances.

After the rather formal discussion of the previous paragraphs we are now prepared to prove the first important mathematical result. 
Lemma 1. Let $\mu$ be the highest weight of a typical Verma module. Then every singular vector $\nu$ in this Verma module is again typical.

Proof. Since singular vectors can just occur in the second factor of $\widehat{\mathrm{sl}}(2)_{-k} \oplus \widehat{\mathrm{sl}}(2)_{k}$ we just have to distinguish two cases, corresponding to the two different signs in (A.8). In the "_"-case one finds

$$
\left(\nu_{1}, \nu_{2}\right)=\left(\mu_{1},-\mu_{2}+2(n k-1)\right) .
$$

The conditions for the existence of a singular vector in the submodule $\nu$ on the other hand read

$$
\begin{aligned}
& -\nu_{1}+\nu_{2}=-\mu_{1}-\mu_{2}-2+2 n k \stackrel{?}{=} 2 l k \\
& -\nu_{1}-\nu_{2}-2=-\mu_{1}+\mu_{2}-2 n k \stackrel{?}{=} 2 l k
\end{aligned}
$$

Each of them could only be satisfied if $\mu$ was atypical. Similar considerations apply to the "+"-case.

Basically the previous Lemma implies that for typical modules the complete structure of singular vectors is captured by the bosonic singular vectors. It should moreover be noted that for $\widehat{\operatorname{psl}}(2 \mid 2)_{k}$ the decoupling conditions for the bosonic roots are precisely those that one obtains in Verma modules over $\widehat{\mathrm{sl}}(2)_{-k-2} \oplus \widehat{\mathrm{sl}}(2)_{k-2}$. As a result we have the

Conjecture 1. The characters of the generalized Fock module based on a typical weight $\mu$ and that of the corresponding irreducible module obtained from the Verma module coincide. In particular the Fock module is irreducible itself.

In order to promote this conjecture to a theorem one would have to discuss the multiplicities of zeroes in the Kac-Kazhdan determinant [67, 68] but we refrain from doing so here. For atypical modules one has to work a bit harder to obtain the characters of irreducible modules, see section 3 for details. We conclude by expressing our expectation that the reasoning of this appendix generalizes to more general classes of affine Lie superalgebras.

\section{References}

[1] O. Aharony, S. S. Gubser, J. M. Maldacena, H. Ooguri and Y. Oz, Large N field theories, string theory and gravity, Phys. Rept. 323 (2000) 183-386 hep-th/9905111. 
[2] R. R. Metsaev and A. A. Tseytlin, Type IIB superstring action in $A d S_{5} \times S^{5}$ background, Nucl. Phys. B533 (1998) 109-126 hep-th/9805028.

[3] J. Rahmfeld and A. Rajaraman, The GS string action on $A d S_{3} \times S^{3}$ with Ramond-Ramond charge, Phys. Rev. D60 (1999) 064014 hep-th/9809164.

[4] N. Berkovits, C. Vafa and E. Witten, Conformal field theory of AdS background with Ramond-Ramond flux, JHEP 03 (1999) 018 hep-th/9902098.

[5] N. Berkovits, M. Bershadsky, T. Hauer, S. Zhukov and B. Zwiebach, Superstring theory on $A d S_{2} \times S^{2}$ as a coset supermanifold, Nucl. Phys. B567 (2000) 61-86 hep-th/9907200.

[6] N. Berkovits, Super-poincare covariant quantization of the superstring, JHEP 04 (2000) 018 hep-th/0001035.

[7] R. Roiban and W. Siegel, Superstrings on $A d S_{5} \times S^{5}$ supertwistor space, JHEP 11 (2000) 024 hep-th/0010104.

[8] M. R. Zirnbauer, Conformal field theory of the integer quantum Hall plateau transition, hep-th/9905054.

[9] M. J. Bhaseen, I. I. Kogan, O. A. Solovev, N. Tanigichi and A. M. Tsvelik, Towards a field theory of the plateau transitions in the Integer Quantum Hall Effect, Nucl. Phys. B580 (2000) 688-720 cond-mat/9912060.

[10] S. Guruswamy, A. LeClair and A. W. W. Ludwig, gl $(N \mid N)$ super-current algebras for disordered Dirac fermions in two dimensions, Nucl. Phys. B583 (2000) 475-512 cond-mat/9909143.

[11] K. B. Efetov, Supersymmetry and theory of disordered metals, Adv. Phys. 32 (1983) 53-127.

[12] D. Bernard, (Perturbed) conformal field theory applied to 2D disordered systems: An introduction, hep-th/9509137.

[13] J. M. Maldacena and H. Ooguri, Strings in $A d S_{3}$ and $S L(2, R)$ WZW model. I, J. Math. Phys. 42 (2001) 2929-2960 hep-th/0001053. 
[14] J. M. Maldacena and H. Ooguri, Strings in $A d S_{3}$ and the $S L(2, R)$ WZW model. III: Correlation functions, Phys. Rev. D65 (2002) 106006 hep-th/0111180.

[15] K. Gawedzki, Noncompact WZW conformal field theories, hep-th/9110076.

[16] J. Teschner, On structure constants and fusion rules in the $S L(2, C) / S U(2)$ WZNW model, Nucl. Phys. B546 (1999) 390-422 [hep-th/9712256].

[17] J. Teschner, Operator product expansion and factorization in the $\mathrm{H}_{3}^{+}$WZNW model, Nucl. Phys. B571 (2000) 555-582 hep-th/9906215.

[18] M. Bershadsky, S. Zhukov and A. Vaintrob, $P S L(n \mid n)$ sigma model as a conformal field theory, Nucl. Phys. B559 (1999) 205-234 hep-th/9902180.

[19] P. Bouwknegt and K. Schoutens, W symmetry in conformal field theory, Phys. Rept. 223 (1993) 183-276 hep-th/9210010].

[20] V. Schomerus and H. Saleur, The GL(1|1) WZW model: From supergeometry to logarithmic CFT, Nucl. Phys. B734 (2006) 221-245 hep-th/0510032.

[21] L. Rozansky and H. Saleur, Quantum field theory for the multivariable Alexander-Conway polynomial, Nucl. Phys. B376 (1992) 461-509.

[22] V. Gurarie, Logarithmic operators in conformal field theory, Nucl. Phys. B410 (1993) 535-549 hep-th/9303160.

[23] M. R. Gaberdiel and H. G. Kausch, A local logarithmic conformal field theory, Nucl. Phys. B538 (1999) 631-658 hep-th/9807091].

[24] M. Flohr, Bits and pieces in logarithmic conformal field theory, Int. J. Mod. Phys. A18 (2003) 4497-4592 hep-th/0111228.

[25] M. R. Gaberdiel, An algebraic approach to logarithmic conformal field theory, Int. J. Mod. Phys. A18 (2003) 4593-4638 hep-th/0111260.

[26] J. S. Caux, I. I. Kogan and A. M. Tsvelik, Logarithmic operators and hidden continuous symmetry in critical disordered models, Nucl. Phys. B466 (1996) 444-462 hep-th/9511134. 
[27] Z. Maassarani and D. Serban, Non-unitary conformal field theory and logarithmic operators for disordered systems, Nucl. Phys. B489 (1997) 603-625 hep-th/9605062.

[28] J. S. Caux, I. Kogan, A. Lewis and A. M. Tsvelik, Logarithmic operators and dynamical extension of the symmetry group in the bosonic SU(2) 0 and SUSY $S U(2)_{2}$ WZNW models, Nucl. Phys. B489 (1997) 469-484 hep-th/9606138.

[29] Y. M. Zou, Categories of finite-dimensional weight modules over type I classical Lie superalgebras, J. Algebra 180 (1996), no. 2 459-482.

[30] J. Germoni, Indecomposable representations of special linear Lie superalgebras, J. Algebra 209 (1998) 367-401.

[31] Y.-Z. Zhang and M. D. Gould, A unified and complete construction of all finite dimensional irreducible representations of gl(2|2), J. Math. Phys. 46 (2005) 013505 math.qa/0405043.

[32] G. Götz, T. Quella and V. Schomerus, Tensor products of psl $(2 \mid 2)$ representations, hep-th/0506072.

[33] V. G. Kac, Lie superalgebras, Adv. Math. 26 (1977) 8-96.

[34] H. Saleur private communication.

[35] L. Rozansky and H. Saleur, $S$ and T matrices for the $U(1 \mid 1)$ WZW model: Application to surgery and three manifolds invariants based on the Alexander-Conway polynomial, Nucl. Phys. B389 (1993) 365-423 hep-th/9203069.

[36] I. Bars, Free fields and new cosets of current algebras, Phys. Lett. B255 (1991) 353-358.

[37] V. G. Kac and M. Wakimoto, Integrable heighest weights modules over affine superalgebras and Appell's function, Comm. Math. Phys. 215 (2001) 631-682 math-ph/0006007.

[38] A. M. Semikhatov, A. Taormina and I. Y. Tipunin, Higher level Appell functions, modular transformations, and characters, Comm. Math. Phys. 255 (2005) 469-512 math.qa/0311314. 
[39] L. Dolan and E. Witten, Vertex operators for $A d S_{3}$ background with Ramond-Ramond flux, JHEP 11 (1999) 003 hep-th/9910205.

[40] D. Gepner and E. Witten, String theory on group manifolds, Nucl. Phys. B278 (1986) 493.

[41] V. S. Dotsenko, Solving the SU(2) conformal field theory with the Wakimoto free field representation, Nucl. Phys. B358 (1991) 547-570.

[42] J. Teschner, On the Liouville three point function, Phys. Lett. B363 (1995) 65-70 hep-th/9507109.

[43] V. G. Kac, Infinite dimensional Lie algebras. Cambridge University Press, Cambridge, 3. ed., 1990.

[44] B. Gerganov, A. LeClair and M. Moriconi, On the beta function for anisotropic current interactions in 2D, Phys. Rev. Lett. 86 (2001) 4753 hep-th/0011189.

[45] A. W. W. Ludwig and K. J. Wiese, The 4-loop beta-function in the $2 D$ non-Abelian Thirring model, and comparison with its conjectured 'exact' form, Nucl. Phys. B661 (2003) 577-607 cond-mat/0211531.

[46] J. L. Cardy, Conformal invariance and statistical mechanics, in Fields, strings and critical phenomena (E. Brézin and J. Zinn-Justin, eds.), Les Houches Summer School, 1988.

[47] F. A. Bais, P. Bouwknegt, M. Surridge and K. Schoutens, Extensions of the Virasoro algebra constructed from Kac-Moody algebras using higher order Casimir invariants, Nucl. Phys. B304 (1988) 348-370.

[48] F. A. Bais, P. Bouwknegt, M. Surridge and K. Schoutens, Coset construction for extended Virasoro algebras, Nucl. Phys. B304 (1988) 371-391.

[49] B. Feigin and E. Frenkel, Quantization of the Drinfeld-Sokolov reduction, Phys. Lett. B246 (1990) 75-81.

[50] E. Frenkel, V. Kac and M. Wakimoto, Characters and fusion rules for $W$ algebras via quantized Drinfeld-Sokolov reductions, Commun. Math. Phys. 147 (1992) 295-328. 
[51] D. Bernard, An introduction to Yangian symmetries, Int. J. Mod. Phys. B7 (1993) 3517-3530 hep-th/9211133.

[52] N. J. MacKay, Introduction to Yangian symmetry in integrable field theory, Int. J. Mod. Phys. A20 (2005) 7189-7218 hep-th/0409183.

[53] I. Bena, J. Polchinski and R. Roiban, Hidden symmetries of the $A d S_{5} \times S^{5}$ superstring, Phys. Rev. D69 (2004) 046002 hep-th/0305116.

[54] L. Dolan, C. R. Nappi and E. Witten, A relation between approaches to integrability in superconformal Yang-Mills theory, JHEP 10 (2003) 017 hep-th/0308089.

[55] B. C. Vallilo, Flat currents in the classical $A d S_{5} \times S^{5}$ pure spinor superstring, JHEP 03 (2004) 037 hep-th/0307018.

[56] M. Hatsuda and K. Yoshida, Classical integrability and super Yangian of superstring on $A d S_{5} \times S^{5}$, Adv. Theor. Math. Phys. 9 (2005) 703-728 hep-th/0407044.

[57] A. Das, A. Melikyan and M. Sato, The algebra of flat currents for the string on $A d S_{5} \times S^{5}$ in the light-cone gauge, JHEP 11 (2005) 015 hep-th/0508183.

[58] D. Kagan and C. A. S. Young, Conformal sigma-models on supercoset targets, Nucl. Phys. B745 (2006) 109-122 hep-th/0512250.

[59] B. H. Miller, Conserved charges in the principal chiral model on a supergroup, JHEP 08 (2006) 010 hep-th/0602006.

[60] M. Bianchi and J. Kluson, Current algebra of the pure spinor superstring in $A d S_{5} \times S^{5}$, JHEP 08 (2006) 030 hep-th/0606188.

[61] B. C. Vallilo, One loop conformal invariance of the superstring in an $A d S_{5} \times S^{5}$ background, JHEP 12 (2002) 042 hep-th/0210064.

[62] N. Berkovits, Quantum consistency of the superstring in $A d S_{5} \times S^{5}$ background, JHEP 03 (2005) 041 hep-th/0411170.

[63] P. A. Grassi, G. Policastro, M. Porrati and P. Van Nieuwenhuizen, Covariant quantization of superstrings without pure spinor constraints, JHEP 10 (2002) 054 hep-th/0112162. 
[64] P. A. Grassi, G. Policastro and P. van Nieuwenhuizen, The quantum superstring as a WZNW model, Nucl. Phys. B676 (2004) 43-63 hep-th/0307056.

[65] P. A. Grassi and P. van Nieuwenhuizen, Gauging cosets, Nucl. Phys. B702 (2004) 189-206 hep-th/0403209.

[66] S. Guttenberg, J. Knapp and M. Kreuzer, On the covariant quantization of type II superstrings, JHEP 06 (2004) 030 hep-th/0405007.

[67] V. Kac and D. Kazhdan, Structure of representations with highest weight of infinite-dimensional Lie algebras., Adv. Math. 34 (1979) 97-108.

[68] V. G. Kac, Highest weight representations of conformal current algebras, in Topological and geometrical methods in field theory (Espoo, 1986), pp. 3-15. World Sci. Publishing, Teaneck, NJ, 1986.

[69] V. G. Kac and D. H. Peterson, Infinite dimensional Lie algebras, theta functions and modular forms, Adv. Math. 53 (1984) 125-264. 